\title{
A Four-Zone Model and Nonlinear Dynamic Analysis of Solution Multiplicity of Buoyancy Ventilation in Underground Building
}

\author{
Yuxing Wang iD and Chunyu Wei \\ School of Architecture, Hunan University, Changsha 410082, Hunan, China \\ Correspondence should be addressed to Yuxing Wang; yuxingwang@hnu.edu.cn
}

Received 2 May 2020; Accepted 26 May 2020; Published 31 August 2020

Guest Editor: Chun-Lai Li

Copyright (c) 2020 Yuxing Wang and Chunyu Wei. This is an open access article distributed under the Creative Commons Attribution License, which permits unrestricted use, distribution, and reproduction in any medium, provided the original work is properly cited.

\begin{abstract}
The solution multiplicity of natural ventilation in buildings is very important to personnel safety and ventilation design. In this paper, a four-zone model of buoyancy ventilation in typical underground building is proposed. The underground structure is divided to four zones, a differential equation is established in each zone, and therefore, there are four differential equations in the underground structure. By solving and analyzing the equilibrium points and characteristic roots of the differential equations, we analyze the stability of three scenarios and obtain the criterions to determine the stability and existence of solutions for two scenarios. According to these criterions, the multiple steady states of buoyancy ventilation in any four-zone underground buildings for different stack height ratios and the strength ratios of the heat sources can be obtained. These criteria can be used to design buoyancy ventilation or natural exhaust ventilation systems in underground buildings. Compared with the two-zone model in (Liu et al. 2020), the results of the proposed four-zone model are more consistent with CFD results in (Liu et al. 2018). In addition, the results of proposed four-zone model are more specific and more detailed in the unstable equilibrium point interval. We find that the unstable equilibrium point interval is divided into two different subintervals corresponding to the saddle point of index 2 and the saddle focal equilibrium point of index 2, respectively. Finally, the phase portraits and vector field diagrams for the two scenarios are given.
\end{abstract}

\section{Introduction}

Nonlinear characteristics exist in systems with various research directions, such as neural network [1-3], chaotic circuit [4-12], and information security [13-15]. Building natural ventilation also has nonlinear characteristics. The solution multiplicity of natural ventilation in buildings is very important to personnel safety and ventilation design [16]. In the last decade, the multisolution problem of natural ventilation in buildings has attracted wide attention. Many papers have been published on the problem of multiple solutions to building natural ventilation [17-23]. These studies can be divided into three categories according to the number of building zones and vents. The first kind research is about the solution multiplicity of single-zone and doubleopening buildings under the combined effect of wind pressure and thermal pressure [19, 24-27]. For example, Heiselberg et al. [24] studied the multiple steady-state properties of single-zone and double-opening buildings under the action of wind pressure and buoyancy through a salt water experiment and CFD simulation; Lishman and Woods [25] reported solution multiplicity in both inclined tunnels and two-story aboveground buildings, and the study focused on the confrontation of wind pressure and buoyancy in a single-story building; Yuan and Glicksman $[19,26]$ researched the effects of different initial conditions on the generation of multiple steady states in single-zone buildings under the combined action of wind pressure and buoyancy; Pulat and Ersan [27] found that different turbulence parameters may produce multiple solutions through CFD simulation. The second kind research is about the solution multiplicity of single-zone and multiple opening buildings [28, 29]. For example, Durrani et al. [28] researched the multiple solutions in a typical aboveground building with one zone and three openings by CFD simulations; through theoretical analysis, Chen and $\mathrm{Li}$ [29] researched the 
buoyancy ventilation of a single-zone building with three horizontal openings. The third kind research is about the solution multiplicity of two-zone buildings [30-33]. For example, Yang et al. [30, 31] analyzed in detail multiple steady states in a two-zone building by theoretical analyses and CFD simulations; Li et al. [32] studied the buoyancy ventilation in a two-story building with two heat sources and three openings by establishing a mathematical model of nonlinear ordinary differential equation; Yang et al. [33] analyzed the smoke exhaust spread of the three entrance tunnel in the fire scenarios and obtained six possible equilibrium states.

Recently, there are some reports on the multiplicity of ventilation solutions of underground structures $[34,35]$. The study of the multiplicity of solutions for the natural ventilation of underground buildings is different from that of overground buildings. Because the underground buildings are not exposed to the outdoor environment, it is mainly thermal pressure ventilation (or buoyancy ventilation) rather than wind pressure ventilation which exist in the under structure. Liu et al. [34] investigated the formation process of multiple steady states in an underground building with two tunnel connecting to the outdoor environment. The heights of two tunnels are same, and one heat source is located in the corner of the buried space. Using the CFD method reproduces the two steady states of the buoyancy ventilation in the underground building. In 2020, Liu et al. [35] made nonlinear dynamic analysis of solution multiplicity of buoyancy ventilation in a typical underground structure. They gave a description of mathematical model of second-order nonlinear differential equation to underground structure with two zones and two tunnel connecting to the outdoor environment. Two heat sources are located in the two zones, respectively, and the heights of the two tunnels are not necessarily the same. By solving the equilibrium points and characteristic roots of the second order differential equation, the solution multiplicity can be determined when the strength ratio of two heat sources and height ratio of two tunnels were changed. However, in the literatures $[34,35]$, the underground structure has only two zones. The deeply burred room and tunnel were treated as one zone, which is just a very simple model. In the real underground structure, the deeply burred room and tunnel are different in temperature, mass flow rate, and air density. So, the deeply burred room and tunnel should not be treated as one zone if a more accurate model is needed. In this paper, we propose a new model, where the buried rooms and tunnels are treated as different zones. Thus, the underground structure consists of four zones, two zones for two tunnels, and two zones for deeply burred room. In this paper, the nomenclature of every variables and constants are shown in abbreviation section.

\section{Proposed New Mathematical Model and Analysis}

In order to study the nonlinear dynamics of typical deepburied underground buildings with two openings, we first established the mathematical mode. Some assumptions were made: (i) each zone is well mixed; (ii) thermal mass is 1; (iii)
$E_{1}>0$; (iv) the mass flow impedance coefficient of the geometry is constant. Because the driving force is from thermal pressure, as illustrated in Figure 1, we divide the building into four zones: we can assume the heat source in the left side as positive, and the heat source of the right side could be either negative or positive, such that we could discuss the scenarios of two heat sources (the heat source in the left and right sides both are positive and the heat source in the left side is positive, the heat source in the right side is negative) and the scenario of one heat source.

2.1. Description of Mathematical Model. The proposed new schematics of underground structure is shown as Figure 1, which contains four zones, zone 1 and zone 2 for deeply burred room and zone 3 and zone 4 for two tunnels. The heights of two tunnels are $H_{1}$ and $H_{2}$ respectively, the $T_{i}, q_{i}(i=1 \sim 4)$ denote the temperature, and mass flow rate of four zones, respectively. Heat sources $E_{1}$ and $E_{2}$ are located in zone 1 and zone 2. As shown in Figure 1, two realizations are considered: for realization 1, the air flow enters from zone 1 to zone 2; for realization 2, the air flow enters from zone 2 to zone 1 .

For realization 1, the heat gain of the internal thermal mass should be equal to the heat released by the heat sources minus the heat loss through airflows. Therefore, we can obtain the heat balance equations of the four zones shown in the following equations:

$$
\begin{aligned}
& M_{1} C_{p} \frac{\mathrm{d} T_{1}}{\mathrm{~d} t}=-q_{1} C_{P}\left(T_{1}-T_{3}\right)+E_{1}, \\
& M_{2} C_{p} \frac{\mathrm{d} T_{2}}{\mathrm{~d} t}=-q_{2} C_{P}\left(T_{2}-T_{1}\right)+E_{2}, \\
& M_{3} C_{p} \frac{\mathrm{d} T_{3}}{\mathrm{~d} t}=-q_{3} C_{P}\left(T_{3}-T_{a}\right), \\
& M_{4} C_{p} \frac{\mathrm{d} T_{4}}{\mathrm{~d} t}=-q_{4} C_{P}\left(T_{4}-T_{2}\right) .
\end{aligned}
$$

According to the flow loop method, the total pressure loss at ventilation vents on the flow loop is balanced by sum of the buoyancy pressure [35]. The pressure balance equation can be obtained as follows:

$$
\begin{gathered}
-\frac{T_{1}-T_{a}}{T_{a}} \rho_{a} g H_{1}+\frac{T_{2}-T_{a}}{T_{a}} \rho_{a} g H_{2} \\
=S_{1} q_{1}^{2}+S_{2} q_{2}^{2}+S_{3} q_{3}^{2}+S_{4} q_{4}^{2} .
\end{gathered}
$$

According to the conservation of mass, we can obtain

$$
q_{1}=q_{2}=q_{3}=q_{4} .
$$

Combining equations (5) and (6), mass flow rate can be obtained as follows:

$$
\begin{aligned}
q_{1} & =q_{2}=q_{3}=q_{4}=q \\
& =\sqrt{\frac{-\left(\left(T_{1}-T_{a}\right) / T_{a}\right) \rho_{a} g H_{1}+\left(\left(T_{2}-T_{a}\right) / T_{a}\right) \rho_{a} g H_{2}}{S_{1}+S_{2}+S_{3}+S_{4}} .}
\end{aligned}
$$




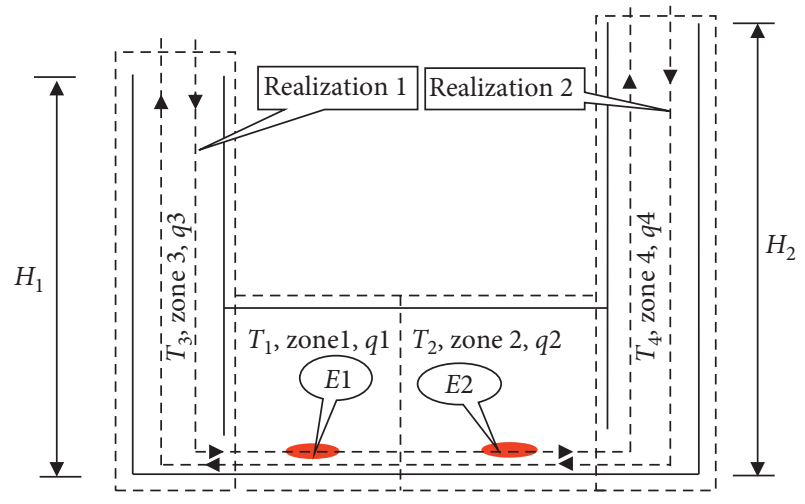

Figure 1: Proposed schematics of four-zone underground structure.

For realization 2, similarly, we can obtain the heat balance equations of the four zones as follows:

$$
\begin{aligned}
& M_{1} C_{p} \frac{\mathrm{d} T_{1}}{\mathrm{~d} t}=-q_{1} C_{P}\left(T_{1}-T_{2}\right)+E_{1}, \\
& M_{2} C_{p} \frac{\mathrm{d} T_{2}}{\mathrm{~d} t}=-q_{2} C_{P}\left(T_{2}-T_{4}\right)+E_{2}, \\
& M_{3} C_{p} \frac{\mathrm{d} T_{3}}{\mathrm{~d} t}=-q_{3} C_{P}\left(T_{3}-T_{1}\right), \\
& M_{4} C_{p} \frac{\mathrm{d} T_{4}}{\mathrm{~d} t}=-q_{4} C_{P}\left(T_{4}-T_{a}\right) .
\end{aligned}
$$

Based on the flow loop method, the total pressure loss at ventilation vents on the flow loop is balanced by sum of the buoyancy pressure, and we can obtain the pressure balance equation as follows:

$$
\frac{T_{1}-T_{a}}{T_{a}} \rho_{a} g H_{1}-\frac{T_{2}-T_{a}}{T_{a}} \rho_{a} g H_{2}=S_{1} q_{1}^{2}+S_{2} q_{2}^{2}+S_{3} q_{3}^{2}+S_{4} q_{4}^{2} .
$$

According to the conservation of mass, we can obtain following equations about air flow rate:

$$
q_{1}=q_{2}=q_{3}=q_{4}=q .
$$

By combining equations (11) and (12), we can obtain

$$
\begin{aligned}
q_{1} & =q_{2}=q_{3}=q_{4} \\
& =\sqrt{\frac{\left(\left(T_{1}-T_{a}\right) / T_{a}\right) \rho_{a} g H_{1}-\left(\left(T_{2}-T_{a}\right) / T_{a}\right) \rho_{a} g H_{2}}{S_{1}+S_{2}+S_{3}+S_{4}} .}
\end{aligned}
$$

2.2. Transformation of Variables and Parameters in Differential Equations. Assuming $k=E_{2} / E_{1}, \quad \Delta T_{1}=T_{1}-T_{a}$, $\Delta T_{2}=T_{2}-T_{\mathrm{a}}, \quad \Delta T_{3}=T_{3}-T_{a}, \quad \Delta T_{4}=T_{4}-T_{a}$, $n=\sqrt{\left(\rho_{a} g H_{1} /\left(S_{1}+S_{2}+S_{3}+S_{4}\right)\right)}, \alpha=H_{2} / H_{1}, M_{1}=M_{2}=M$, $M_{3}=M_{4}=M / \xi$, and $\tau=t /\left(M C_{p}\right)$, we can obtain simplified nonlinear differential equations.
For realization 1, according to equations (1) (4), (7) and above assumptions about variables and parameters, the simplified differential equations can be obtained as follows:

$$
\begin{aligned}
& f_{1}=\frac{\mathrm{d} \Delta T_{1}}{\mathrm{~d} \tau}=-n \sqrt{\alpha \Delta T_{2}-\Delta T_{1}}\left(\Delta T_{1}-\Delta T_{3}\right)+E_{1}, \\
& f_{2}=\frac{\mathrm{d} \Delta T_{2}}{\mathrm{~d} \tau}=-n \sqrt{\alpha \Delta T_{2}-\Delta T_{1}}\left(\Delta T_{2}-\Delta T_{1}\right)+E_{2}, \\
& f_{3}=\frac{\mathrm{d} \Delta T_{3}}{\mathrm{~d} \tau}=-\xi n \sqrt{\alpha \Delta T_{2}-\Delta T_{1}} \Delta T_{3}, \\
& f_{4}=\frac{\mathrm{d} \Delta T_{4}}{\mathrm{~d} \tau}=-\xi n \sqrt{\alpha \Delta T_{2}-\Delta T_{1}}\left(\Delta T_{4}-\Delta T_{2}\right) .
\end{aligned}
$$

For realization 2, according to equations (8) (11) and (14) and above assumptions about variables and parameters, the simplified differential equations can be obtained as follows:

$$
\begin{aligned}
& f_{1}=\frac{\mathrm{d} \Delta T_{1}}{\mathrm{~d} \tau}=-n \sqrt{\Delta T_{1}-\alpha \Delta T_{2}}\left(\Delta T_{1}-\Delta T_{2}\right)+E_{1}, \\
& f_{2}=\frac{\mathrm{d} \Delta T_{2}}{\mathrm{~d} \tau}=-n \sqrt{\Delta T_{1}-\alpha \Delta T_{2}}\left(\Delta T_{2}-\Delta T_{4}\right)+E_{2}, \\
& f_{3}=\frac{\mathrm{d} \Delta T_{3}}{\mathrm{~d} \tau}=-\xi n \sqrt{\Delta T_{1}-\alpha \Delta T_{2}}\left(\Delta T_{3}-\Delta T_{1}\right), \\
& f_{4}=\frac{\mathrm{d} \Delta T_{4}}{\mathrm{~d} \tau}=-\xi n \sqrt{\Delta T_{1}-\alpha \Delta T_{2}} \Delta T_{4} .
\end{aligned}
$$

2.3. Nonlinear Dynamic Analysis. We can assume the heat source in the left side as positive and the heat source of the right side could be either negative or positive, such that we could discuss the scenarios of two heat sources (the heat source in the left and right sides both are positive and the heat source in the left side is positive, the heat source in the right side is negative) and the scenario of one heat source.

2.3.1. Stability Analysis for Scenario 1 ( $k$ Is Fixed and $\alpha$ Is Control Parameter). Here, two conditions $k>0$ (the heat source in the left and right sides both are positive) and $k<0$ (the heat source in the left side is positive, and the heat source in the right side is negative) are discussed at the same time. We should solve equilibrium points and characteristic roots before stability analysis:

(1) Analysis of Equilibrium Points and Characteristic Roots for Realization 1. The steady state solutions (equilibrium points) of realization 1 equations (15) (18) are denoted as $\left(\Delta T_{01}, \Delta T_{02}, \Delta T_{03}, \Delta T_{04}\right)$. In order to solve the equilibrium points, we can make that the right sides of equations (15) (18) equal to zero; and the following equations hold: 


$$
\begin{array}{r}
-n \sqrt{\alpha \Delta T_{2}-\Delta T_{1}}\left(\Delta T_{1}-\Delta T_{3}\right)+E_{1}=0, \\
-n \sqrt{\alpha \Delta T_{2}-\Delta T_{1}}\left(\Delta T_{2}-\Delta T_{1}\right)+E_{2}=0, \\
-\xi n \sqrt{\alpha \Delta T_{2}-\Delta T_{1}} \Delta T_{3}=0, \\
-\xi n \sqrt{\alpha \Delta T_{2}-\Delta T_{1}}\left(\Delta T_{4}-\Delta T_{2}\right)=0 .
\end{array}
$$

By solving equations (23) (26), we can obtain the equilibrium points as follows:

$$
\begin{aligned}
\Delta T_{01} & =\frac{E_{1}}{n^{2 / 3}\left(-E_{1}+E_{1} \alpha+E_{2} \alpha\right)^{1 / 3}}=\frac{E_{1}^{2 / 3}}{n^{2 / 3}(\alpha k-1+\alpha)^{1 / 3}}, \\
\Delta T_{02} & =\Delta T_{04}=(1+k) \Delta T_{01} \\
& =\frac{(1+k) E_{1}}{n^{2 / 3}\left(-E_{1}+E_{1} \alpha+E_{2} \alpha\right)^{1 / 3}}=\frac{(1+k) E_{1}^{2 / 3}}{n^{2 / 3}(\alpha k-1+\alpha)^{1 / 3}},
\end{aligned}
$$

$$
\Delta T_{03}=0
$$

According to the characteristic determinant equal to 0 , the solution of the differential equation can be discussed. The characteristic determinant equation is shown as follows. The expression of characteristic determinant is that

$$
|J(Q)-\lambda E|=\left|\begin{array}{cccc}
\frac{\partial f_{1}}{\partial \Delta T_{1}}-\lambda & \frac{\partial f_{1}}{\partial \Delta T_{2}} & \frac{\partial f_{1}}{\partial \Delta T_{3}} & \frac{\partial f_{1}}{\partial \Delta T_{4}} \\
\frac{\partial f_{2}}{\partial \Delta T_{1}} & \frac{\partial f_{2}}{\partial \Delta T_{2}}-\lambda & \frac{\partial f_{2}}{\partial \Delta T_{3}} & \frac{\partial f_{2}}{\partial \Delta T_{4}} \\
\frac{\partial f_{3}}{\partial \Delta T_{1}} & \frac{\partial f_{3}}{\partial \Delta T_{2}} & \frac{\partial f_{3}}{\partial \Delta T_{3}}-\lambda & \frac{\partial f_{3}}{\partial \Delta T_{4}} \\
\frac{\partial f_{4}}{\partial \Delta T_{1}} & \frac{\partial f_{4}}{\partial \Delta T_{2}} & \frac{\partial f_{4}}{\partial \Delta T_{3}} & \frac{\partial f_{4}}{\partial \Delta T_{4}}-\lambda
\end{array}\right|=0 .
$$

Combining equations (15) (18), we can obtain the following expression:

$$
\left|\begin{array}{cccc}
\frac{n\left(\Delta T_{01}-\Delta T_{03}\right)}{2 A}-n A-\lambda & -\frac{n \alpha\left(\Delta T_{01}-\Delta T_{03}\right)}{2 A} & n A & 0 \\
\frac{n\left(\Delta T_{02}-\Delta T_{01}\right)}{2 A}+n A & -\frac{n \alpha\left(\Delta T_{02}-\Delta T_{01}\right)}{2 A}-n A-\lambda & 0 & 0 \\
\frac{\xi n \Delta T_{03}}{2 A} & -\frac{\xi n \alpha \Delta T_{03}}{2 A} & -\xi n A-\lambda & 0 \\
\frac{\xi n\left(\Delta T_{04}-\Delta T_{02}\right)}{2 A} & -\frac{\xi n \alpha\left(\Delta T_{04}-\Delta T_{02}\right)}{2 A}+\xi n A & 0 & -\xi n A-\lambda
\end{array}\right|=0
$$

where $J(Q)$ is the Jacobian matrix of equilibrium point $Q, \lambda$ is the characteristic root, $E$ is the identity matrix, and

$$
\begin{aligned}
& A=\sqrt{\alpha \Delta T_{02}-\Delta T_{01}}=\frac{(\alpha k-1+\alpha)^{1 / 3} E_{1}^{1 / 3}}{n^{1 / 3}}, \\
& B=\Delta T_{01}-\Delta T_{03}=\frac{E_{1}^{2 / 3}}{n^{2 / 3}(\alpha k-1+\alpha)^{1 / 3}}, \\
& C=\Delta T_{02}-\Delta T_{01}=\frac{k E_{1}^{2 / 3}}{n^{2 / 3}(\alpha k-1+\alpha)^{1 / 3}} .
\end{aligned}
$$

$$
\begin{aligned}
\lambda_{3,4} & =\frac{-b \pm \sqrt{b^{2}-4 a c}}{2 a} \\
& =\frac{-(n(\alpha C-B) / 2 A)-2 n A \pm \sqrt{[(n(\alpha C-B) / 2 A)+2 n A]^{2}-4 n^{2}\left[(\alpha(B+C) / 2)+A^{2}-(B / 2)\right]}}{2},
\end{aligned}
$$
solution exists for equations (27) and (28), we have

$$
\alpha k-1+\alpha>0 \text {. }
$$
to a quadratic formula:

Assuming $k=E_{2} / E_{1}, \alpha=H_{2} / H_{1}$, to ensure that a real

Two other characteristic roots can be obtained according 
where

$$
\left\{\begin{array}{l}
a=1 ; \\
b=\frac{n(\alpha C-B)}{2 A}+2 n A=\frac{n^{2 / 3} E_{1}^{1 / 3}(5 \alpha k-5+4 \alpha)}{2(\alpha k-1+\alpha)^{2 / 3}}, \\
c=n^{2}\left[\frac{\alpha(B+C)}{2}+A^{2}-\frac{B}{2}\right]=\frac{3 E_{1}^{2 / 3}(\alpha k-1+\alpha)^{2 / 3} n^{4 / 3}}{2}>0, \\
\Delta=b^{2}-4 a c=\frac{n^{4 / 3} E_{1}^{2 / 3}(5 \alpha k-5+4 \alpha)^{2}}{4(\alpha k-1+\alpha)^{4 / 3}}-6 E_{1}^{2 / 3}(\alpha k-1+\alpha)^{2 / 3} n^{4 / 3} .
\end{array}\right.
$$

For convenience, the characteristic roots are set as follows: the real root are $\gamma_{i}(i=1,2,3,4)$; the complex roots are $\sigma_{i}+j \omega_{i}(i=1,2,3,4)$. It is easy to know that $\lambda_{1,2}$ are always real number, $\lambda_{1}=\gamma_{1}=\lambda_{2}=\gamma_{2}$.

If the discriminant $\Delta$ is greater than 0 , both characteristic roots are real number, namely, $\lambda_{3}=\gamma_{3}$ and $\lambda_{4}=\gamma_{4}$. From equations (37) and (38), we can know that when $\Delta>0$, the following expression holds:

$$
n^{4 / 3} E_{1}^{2 / 3}(5 \alpha k-5+4 \alpha)^{2}>24 n^{4 / 3} E_{1}^{2 / 3}(\alpha k-1+\alpha)^{2} .
$$

It is clear that

$$
|5 \alpha k-5+4 \alpha|>|2 \sqrt{6}(\alpha k-1+\alpha)| .
$$

When the discriminant $\Delta$ is less than 0 , conjugate complex roots exist, namely, $\lambda_{3,4}=\sigma_{3,4} \pm j \omega_{3,4}$. From equations (37) and (38), we can know that when $\Delta<0$, the following expression holds:

$$
|5 \alpha k-5+4 \alpha|<|2 \sqrt{6}(\alpha k-1+\alpha)| .
$$

(2) Stability Analysis for Realization 1 ( $k$ Is Fixed and $\alpha$ Is Control Parameter)

(1) No equilibrium point exists in realization 1

If no equilibrium point exists in realization 1 , from equation (36), it is known that the following expression is satisfied:

$$
\alpha k-1+\alpha<0 .
$$

Therefore from expression (42), when $k<-1$, it is clear that $\alpha>(1 /(k+1))$ and when $k>-1$, we know that $0<\alpha<(1 /(k+1))$.

(2) A stable equilibrium point exists in realization 1

(2.1) Assuming there are all negative real characteristic roots

In this case, $\lambda_{1}, \lambda_{2}, \lambda_{3}$, and $\lambda_{4}$ are all less than 0 , and the system has a stable solution.

We know that owing to $\alpha k-1+\alpha>0$, from equation (35), it is clear that $\lambda_{1}$ and $\lambda_{2}$ are always less than 0 . To make $\lambda_{3,4}<0$, according to Vieta theorem, we know that $\lambda_{3}+\lambda_{4}=-b$ and $\lambda_{3} \lambda_{4}=c$. From equation (38), it is known that always $c>0$, and owing to $\alpha k-1+\alpha>0$, from equation (38), if $5 \alpha k-5+4 \alpha>0$, we have $-b<0$, then $\lambda_{3,4}<0$. In this case, the absolute value sign in equation (40) can be removed; therefore, the following expression (43) can be obtained:

$$
5 \alpha k-5+4 \alpha>2 \sqrt{6}(\alpha k-1+\alpha) .
$$

From expression (43), we can obtain the following expression:

$$
\alpha[k(5-2 \sqrt{6})+4-2 \sqrt{6}]>5-2 \sqrt{6} .
$$

In summary, if there are all negative real characteristic roots, the system is stable when the following inequalities are satisfied:

$$
\left\{\begin{array}{l}
\alpha(k+1)-1>0, \\
\alpha(5 k+4)-5>0, \\
\alpha[k(5-2 \sqrt{6})+4-2 \sqrt{6}]>5-2 \sqrt{6} .
\end{array}\right.
$$

(2.2) Assuming two negative real roots and two conjugate complex roots

In this case, the four characteristic roots behave as $\lambda_{1}=\gamma_{1}=\lambda_{2}=\gamma_{2}, \lambda_{3,4}=\sigma_{3,4} \pm j \omega_{3,4}$, where $\lambda_{1}$ and $\lambda_{2}$ are two negative real roots and $\lambda_{3}, \lambda_{4}$ are two conjugate complex roots. When $\lambda_{1}, \lambda_{2}, \sigma_{3}$, and $\sigma_{4}$ are all less than 0 , the system has a stable solution.

From equation (37), we know $\sigma_{3,4}=-b / 2$. Owing to $\lambda_{1,2}<0$, to make $\sigma_{3,4}<0$, we need $-b<0$, and from equation (38), we can obtain $5 \alpha k+4 \alpha-5>0$.

In this case, the absolute value sign in expression (41) can be removed; therefore, the following expression can be obtained:

$$
5 \alpha k-5+4 \alpha<2 \sqrt{6}(\alpha k-1+\alpha) .
$$

From (46), we can obtain that

$$
\alpha[k(5-2 \sqrt{6})+4-2 \sqrt{6}]<5-2 \sqrt{6} .
$$

In summary, $\lambda_{1}, \lambda_{2}, \sigma_{3}$, and $\sigma_{4}$ are all less than 0 , the system is stable when the following inequalities are satisfied:

$$
\left\{\begin{array}{l}
\alpha(k+1)-1>0, \\
\alpha(5 k+4)-5>0, \\
\alpha[k(5-2 \sqrt{6})+4-2 \sqrt{6}]<5-2 \sqrt{6}
\end{array}\right.
$$


Combining expressions (45) and (48), a stable equilibrium point exists in realization 1 , when the following expression is satisfied:

$$
\left\{\begin{array}{l}
\alpha(k+1)-1>0, \\
\alpha(5 k+4)-5>0
\end{array}\right.
$$

Case 1: when $k>-4 / 5$, it is easy to know that $5 k+4>0$ and $k+1>0$, then expression (49) can be simplified to

$$
\left\{\begin{array}{l}
\alpha>\frac{1}{k+1}, \\
\alpha>\frac{5}{5 k+4} .
\end{array}\right.
$$

From $5 k+4>0$ and $k+1>0$, it is clear that $(1 /(k+1))-(5 /(5 k+4))<0$ and $0<(1 /(k+1))<$ $(5 /(5 k+4))$,

Therefore, from expression (49), it can be known that, when $k>-(4 / 5), \alpha>(5 /(5 k+4))$

Case 2: when $-1<k<-(4 / 5)$, it is easy to know that $5 k+4<0$ and $k+1>0$.

Expression (49) can be simplified to

$$
\left\{\begin{array}{l}
\alpha>\frac{1}{k+1} \\
\alpha<\frac{5}{5 k+4}
\end{array}\right.
$$

Owing to $5 k+4<0$ and $k+1>0$, it is clear that $(5 /(5 k+4))<0$ and $(1 /(k+1))>0$. Therefore, when $-1<k<-(4 / 5), \alpha$ does not exist.

Case 3: when $k<-1$, it is easy to know that $5 k+4<0$ and $k+1<0$.

Expression (49) can be simplified to

$$
\left\{\begin{array}{l}
\alpha<\frac{1}{k+1}, \\
\alpha<\frac{5}{5 k+4} .
\end{array}\right.
$$

Owing to $5 k+4<0$ and $k+1<0$, it is clear that $(1 /(k+1))-(5 /(5 k+4))<0$. Thus we can obtain that $(5 /(5 k+4))<(1 /(k+1))<0$. Therefore, when $k<-1, \alpha$ does not exist.

In conclusion, we can obtain following several cases:

Case 1: $k>-(4 / 5)$ and $\alpha>(5 /(5 k+4))$

Case 2: $-1<k<-(4 / 5)$ and $\alpha$ does not exist

Case 3: $k<-1$ and $\alpha$ does not exist

(3) An unstable equilibrium point exists in realization 1 (3.1) Assuming characteristic roots are all real and at least one is a positive
As long as one characteristic root is greater than 0 , the equilibrium point of the system is unstable.

Owing to $\alpha k-1+\alpha>0, \lambda_{1}$ and $\lambda_{2}$ are always less than 0 and only $\lambda_{3}$ or $\lambda_{4}$ is greater than 0 . According to Vieta theorem, $\lambda_{3}+\lambda_{4}=-b$ and $\lambda_{3} \lambda_{4}=c$; it is known that $c>0$, and owing to $\alpha k-1+\alpha>0$, from equation (38), if $5 \alpha k-5+4 \alpha<0$, we can obtain $-b>0$, then $\lambda_{3,4}>0$.

In this case, the number in the left absolute value sign of expression (40) is less than zero, right absolute value sign of equation (40) is greater than zero, the absolute value signs are removed, and expression (40) can be transformed as follows:

$$
5-4 \alpha-5 \alpha k>2 \sqrt{6}(\alpha k-1+\alpha) .
$$

From expression (53), we can obtain

$$
\alpha[k(2 \sqrt{6}+5)+2 \sqrt{6}+4]<5+2 \sqrt{6}
$$

In summary, $\lambda_{1}$ and $\lambda_{2}$ are less than 0 and $\lambda_{3}$ and $\lambda_{4}$ are greater than 0 . The system equilibrium point is unstable, and it is the saddle point of index 2, when the following inequalities are satisfied:

$$
\left\{\begin{array}{l}
\alpha k+\alpha-1>0, \\
5 \alpha k+4 \alpha-5<0 \\
\alpha[k(2 \sqrt{6}+5)+2 \sqrt{6}+4]<5+2 \sqrt{6}
\end{array}\right.
$$

Firstly, we consider the coefficient of $\alpha$ on the left side of third equation in expression (55) is greater than 0 , namely, $k(2 \sqrt{6}+5)+2 \sqrt{6}+4>0$, we can obtain $k>((-2 \sqrt{6}-4) /(2 \sqrt{6}+5))$.

Then, we consider the coefficient of $\alpha$ on the left side of the third equation in expression (55) is less than 0 , namely, $k(2 \sqrt{6}+5)+2 \sqrt{6}+4<0$, we can obtain $k<((-2 \sqrt{6}-4) /(2 \sqrt{6}+5))$.

It is easy to know that $-1<((-2 \sqrt{6}-$ $4) /(2 \sqrt{6}+5))<-(4 / 5)$. We discuss following several cases:

Case 1: when $((-2 \sqrt{6}-4) /(2 \sqrt{6}+5))<k<-(4 / 5)$, owing to $-1<((-2 \sqrt{6}-4) /(2 \sqrt{6}+5))<-(4 / 5)$, it is easy to know that $5 k+4<0, \quad k+1>0$ and $k(2 \sqrt{6}+5)+2 \sqrt{6}+4>0$. Expression (55) is simplified as

$$
\left\{\begin{array}{l}
\alpha>\frac{1}{k+1}, \\
\alpha>\frac{5}{5 k+4}, \\
\alpha<\frac{5+2 \sqrt{6}}{k(2 \sqrt{6}+5)+2 \sqrt{6}+4} .
\end{array}\right.
$$


From $5 k+4<0, k+1>0, k(2 \sqrt{6}+5)+2 \sqrt{6}+4>0$, it is clear that $(1 /(k+1))-((5+2 \sqrt{6}) /(k(2 \sqrt{6}+5)+$ $2 \sqrt{6}+4))<0$ and $(5 /(5 k+4))-(1 /(k+1))<0$. So we have $\quad 0<(1 /(k+1))<((5+2 \sqrt{6}) /(k(2 \sqrt{6}+5)+$ $2 \sqrt{6}+4))$ and $(5 /(5 k+4))<0$.

Therefore, when $((-2 \sqrt{6}-4) /(2 \sqrt{6}+5))<k<-$ $(4 / 5)$, it can be obtained that $(1 /(k+1))<\alpha<((2 \sqrt{6}+$ $5) /(k(2 \sqrt{6}+5)+2 \sqrt{6}+4))$.

Case 2: when $-(4 / 5)<k$, owing to $-1<((-2 \sqrt{6}-4) /(2 \sqrt{6}+5))<-(4 / 5)$, it is easy to know that $5 k+4>0, \quad k+1>0$ and $k(2 \sqrt{6}+$ $5)+2 \sqrt{6}+4>0$. Expression (55) can be simplified to

$$
\left\{\begin{array}{l}
\alpha>\frac{1}{k+1}, \\
\alpha<\frac{5}{5 k+4}, \\
\alpha<\frac{5+2 \sqrt{6}}{k(2 \sqrt{6}+5)+2 \sqrt{6}+4} .
\end{array}\right.
$$

From $5 k+4>0, k+1>0, k(2 \sqrt{6}+5)+2 \sqrt{6}+4>0$, it is clear that $(5 /(5 k+4))-((5+2 \sqrt{6}) /(k(2 \sqrt{6}+5)+2$ $\sqrt{6}+4))>0$ and $((5+2 \sqrt{6}) /(k(2 \sqrt{6}+5)+2 \sqrt{6}+4))$ $-(1 /(k+1))>0$. So we have $0<(1 /(k+1))<((5+2$ $\sqrt{6}) /(k(2 \sqrt{6}+5)+2 \sqrt{6}+4))<(5 /(5 k+4))$. Therefore, when $-(4 / 5)<k$, we have $(1 /(k+1))<\alpha<((2 \sqrt{6}+5) /$ $(k(2 \sqrt{6}+5)+2 \sqrt{6}+4))$.

Case 3: when $-1<k<((-2 \sqrt{6}-4) /(2 \sqrt{6}+5))$, owing to $-1<((-2 \sqrt{6}-4) /(2 \sqrt{6}+5))<-(4 / 5)$, it is easy to know that $5 k+4<0, \quad k+1>0$, and $k(2 \sqrt{6}+5)+$ $2 \sqrt{6}+4<0$.

Expression (55) can be simplified to

$$
\left\{\begin{array}{l}
\alpha>\frac{1}{k+1}, \\
\alpha>\frac{5}{5 k+4}, \\
\alpha>\frac{5+2 \sqrt{6}}{k(2 \sqrt{6}+5)+2 \sqrt{6}+4} .
\end{array}\right.
$$

From $5 k+4<0, k+1>0, k(2 \sqrt{6}+5)+2 \sqrt{6}+4<0$, it is easy to know that $((5+2 \sqrt{6}) /(k(2 \sqrt{6}+$ $5)+2 \sqrt{6}+4))-(5 /(5 k+4))<0$, so we have $((5+$ $2 \sqrt{6}) /(k(2 \sqrt{6}+5)+2 \sqrt{6}+4))<(5 /(5 k+4))<0$ and $0<(1 /(k+1))$

Therefore, when $-1<k<((-2 \sqrt{6}-4) /(2 \sqrt{6}+5))$, we can obtain that $(1 /(k+1))<\alpha$.
Case 4: when $k<-1$, owing to $-1<((-2 \sqrt{6}-4) /(2 \sqrt{6}+5))<-(4 / 5)$, it is easy to know that $5 k+4<0, \quad k+1<0$, and $k(2 \sqrt{6}+$ $5)+2 \sqrt{6}+4<0$. Equation (55) can be simplified to

$$
\left\{\begin{array}{l}
\alpha<\frac{1}{k+1}, \\
\alpha>\frac{5}{5 k+4}, \\
\alpha>\frac{5+2 \sqrt{6}}{k(2 \sqrt{6}+5)+2 \sqrt{6}+4} .
\end{array}\right.
$$

From $\quad 5 k+4<0, \quad k+1<0 \quad$ and $\quad k(2 \sqrt{6}+$ $5)+2 \sqrt{6}+4<0$, it is clear that $((5+2 \sqrt{6}) /(k(2 \sqrt{6}+$ $5)+2 \sqrt{6}+4))-(5 /(5 k+4))<0$ and $(1 /(k+1))-$ $((5+2 \sqrt{6}) /(k(2 \sqrt{6}+5)+2 \sqrt{6}+4))<0$, so we have $(1 /(k+1))<((5+2 \sqrt{6}) /(k(2 \sqrt{6}+5)+2 \sqrt{6}+4))<$ $(5 /(5 k+4))<0$. Therefore, when $k<-1, \alpha$ does not exist.

From what has been discussed above, we can obtain following relation between $k$ and $\alpha$ :

Case 1: $\quad((-2 \sqrt{6}-4) /(2 \sqrt{6}+5))<k<-(4 / 5)$ and $(1 /(k+1))<\alpha<((2 \sqrt{6}+5) /(k(2 \sqrt{6}+5)+2 \sqrt{6}+4))$

Case 2: $-(4 / 5)<k$ and $(1 /(k+1))<\alpha<((2 \sqrt{6}+5) /$ $(k(2 \sqrt{6}+5)+2 \sqrt{6}+4))$

Case 3: $\quad-1<k<((-2 \sqrt{6}-4) /(2 \sqrt{6}+5)) \quad$ and $(1 /(k+1))<\alpha$

Case 4: $k<-1$ and $\alpha$ does not exist.

(3.2) Assuming two negative real roots and two conjugate complex roots

In this case, the four characteristic roots behave as $\lambda_{1}=\gamma_{1}=\lambda_{2}=\gamma_{2}, \lambda_{3,4}=\sigma_{3,4} \pm j \omega_{3,4}$, where $\lambda_{1}$ and $\lambda_{2}$ are two negative real roots and $\lambda_{3}$ and $\lambda_{4}$ are two conjugate complex roots.

Owing to $\lambda_{1}$ and $\lambda_{2}$ are always less than 0 , when $\sigma_{3,4}$ is greater than 0 , the equilibrium point of the system is unstable and it is the saddle-focus point of index 2 . From (37), we know $\sigma_{3,4}=-b / 2$. To make $\sigma_{3,4}>0$, we need $-b>0$, and from equation (38), we can obtain $5 \alpha k+4 \alpha-5<0$.

In this case, the absolute value sign in Expression (41) can be removed; therefore, the following expression can be obtained:

$$
5-5 \alpha k-4 \alpha<2 \sqrt{6}(\alpha k-1+\alpha) .
$$

From expression (60), we can obtain that

$$
\alpha[k(5+2 \sqrt{6})+4+2 \sqrt{6}]>2 \sqrt{6}+5
$$


In summary, $\lambda_{1}$ and $\lambda_{2}$ are less than $0, \sigma_{3}$ and $\sigma_{4}$ are greater than 0 , the system equilibrium point is unstable, and it is the saddle-focus point of index 2, when the following inequalities are satisfied:

$$
\left\{\begin{array}{l}
\alpha(k+1)-1>0, \\
\alpha(5 k+4)-5<0 \\
\alpha[k(5+2 \sqrt{6})+4+2 \sqrt{6}]>2 \sqrt{6}+5
\end{array}\right.
$$

According to above discussion, we can obtain the following several cases:

Case 1: $\quad((-4-2 \sqrt{6}) /(5+2 \sqrt{6}))<k<-(4 / 5)$ and $\alpha>((5+2 \sqrt{6}) /(k(5+2 \sqrt{6})+4+2 \sqrt{6}))$

Case 2: $-(4 / 5)<k$ and $((5+2 \sqrt{6}) /(k(5+2 \sqrt{6})+4+$ $2 \sqrt{6}))<\alpha<(5 /(5 k+4))$

Case 3: $-1<k<((-4-2 \sqrt{6}) /(5+2 \sqrt{6}))$ and $\alpha$ does not exist

Case 4: $k<-1$ and $\alpha$ does not exist

If $k$ and $\alpha$ satisfy the above relation, the system equilibrium point is the saddle-focus point of index 2 , which is unstable.

(3) Analysis of Equilibrium Points and Characteristic Roots for Realization 2. The steady state solution (equilibrium points) of realization 2, equations (19) (22), is denoted as $\left(\Delta T_{01}, \Delta T_{02}, \Delta T_{03}, \Delta T_{04}\right)$. In order to solve the equilibrium points, we can make that the right sides of equations (19) (22) equal to zero; the following equations hold:

$$
\begin{gathered}
-n \sqrt{\Delta T_{1}-\alpha \Delta T_{2}}\left(\Delta T_{1}-\Delta T_{2}\right)+E_{1}=0, \\
-n \sqrt{\Delta T_{1}-\alpha \Delta T_{2}}\left(\Delta T_{2}-\Delta T_{4}\right)+E_{2}=0, \\
-\xi n \sqrt{\Delta T_{1}-\alpha \Delta T_{2}}\left(\Delta T_{3}-\Delta T_{1}\right)=0,
\end{gathered}
$$

$$
-\xi n \sqrt{\Delta T_{1}-\alpha \Delta T_{2}} \Delta T_{4}=0 .
$$

By solving equations (63) (66), we can obtain the equilibrium points as follows:

$$
\begin{aligned}
\Delta T_{02} & =\frac{E_{2}}{n^{2 / 3}\left(E_{2}+E_{1}-E_{1} \alpha k\right)^{1 / 3}}=\frac{k E_{1}^{2 / 3}}{n^{2 / 3}(k+1-\alpha k)^{1 / 3}}, \\
\Delta T_{01} & =\Delta T_{03}=\frac{1+k}{k} \Delta T_{02}=\frac{(1+k) E_{1}}{n^{2 / 3}\left(E_{2}+E_{1}-E_{1} \alpha k\right)^{1 / 3}} \\
& =\frac{(1+k) E_{1}^{2 / 3}}{n^{2 / 3}(k+1-\alpha k)^{1 / 3}}, \\
\Delta T_{04} & =0 .
\end{aligned}
$$

According to the characteristic determinant equal to 0 , the solution of the differential equation can be discussed. The characteristic determinant equation is that

$$
|J(Q)-\lambda E|=\left|\begin{array}{cccc}
\frac{\partial f_{1}}{\partial \Delta T_{1}}-\lambda & \frac{\partial f_{1}}{\partial \Delta T_{2}} & \frac{\partial f_{1}}{\partial \Delta T_{3}} & \frac{\partial f_{1}}{\partial \Delta T_{4}} \\
\frac{\partial f_{2}}{\partial \Delta T_{1}} & \frac{\partial f_{2}}{\partial \Delta T_{2}}-\lambda & \frac{\partial f_{2}}{\partial \Delta T_{3}} & \frac{\partial f_{2}}{\partial \Delta T_{4}} \\
\frac{\partial f_{3}}{\partial \Delta T_{1}} & \frac{\partial f_{3}}{\partial \Delta T_{2}} & \frac{\partial f_{3}}{\partial \Delta T_{3}}-\lambda & \frac{\partial f_{3}}{\partial \Delta T_{4}} \\
\frac{\partial f_{4}}{\partial \Delta T_{1}} & \frac{\partial f_{4}}{\partial \Delta T_{2}} & \frac{\partial f_{4}}{\partial \Delta T_{3}} & \frac{\partial f_{4}}{\partial \Delta T_{4}}-\lambda
\end{array}\right|=0 .
$$

Combining equations (19) (22), we can obtain the following expression as follows:

$$
\begin{aligned}
& \left|\begin{array}{cccc}
-\frac{n\left(\Delta T_{01}-\Delta T_{02}\right)}{2 A}-n A-\lambda & \frac{n \alpha\left(\Delta T_{01}-\Delta T_{02}\right)}{2 A}+n A & 0 & 0 \\
-\frac{n\left(\Delta T_{02}-\Delta T_{04}\right)}{2 A} & \frac{n \alpha\left(\Delta T_{02}-\Delta T_{04}\right)}{2 A}-n A-\lambda & 0 & n A \\
-\frac{\xi n\left(\Delta T_{03}-\Delta T_{01}\right)}{2 A}+\xi n A & \frac{\xi n \alpha\left(\Delta T_{03}-\Delta T_{01}\right)}{2 A} & -\xi n A-\lambda & 0 \\
-\frac{\xi n \Delta T_{04}}{2 A} & \frac{\xi n \alpha \Delta T_{04}}{2 A} & 0 & -\xi n A-\lambda
\end{array}\right|=0 \text {, } \\
& A=\sqrt{\Delta T_{01}-\alpha \Delta T_{02}}=\frac{(k+1-\alpha k)^{1 / 3} E_{1}^{1 / 3}}{n^{1 / 3}}, \\
& B=\Delta T_{01}-\Delta T_{02}=\frac{E_{1}^{2 / 3}}{n^{2 / 3}(k+1-\alpha k)^{1 / 3}}, \\
& C=\Delta T_{02}-\Delta T_{04}=\frac{k E_{1}^{2 / 3}}{n^{2 / 3}(k+1-\alpha k)^{1 / 3}},
\end{aligned}
$$


Combining equations (71) (74), we can obtain characteristic roots as follows:

$$
\begin{aligned}
\lambda_{1} & =\lambda_{2}=-\xi n A=-\xi n \sqrt{\Delta T_{01}-\alpha \Delta T_{02}} \\
& =-\xi n^{2 / 3} E_{1}^{1 / 3}(k+1-\alpha k)^{1 / 3} .
\end{aligned}
$$

$$
\begin{aligned}
\lambda_{3,4} & =\frac{-b \pm \sqrt{b^{2}-4 a c}}{2 a} \\
& =\frac{-(n(B-\alpha C) / 2 A)-2 n A \pm \sqrt{[(n(B-\alpha C) / 2 A)+2 n A]^{2}-4 n^{2}\left[((B+C) / 2)+A^{2}-(\alpha C / 2)\right]}}{2},
\end{aligned}
$$

To ensure that a real solution exists for equations (67) and (68), we have

$$
k+1-\alpha k>0 .
$$

Two other characteristic roots can be obtained according to the quadratic formula: where

$$
\left\{\begin{array}{l}
a=1, \\
b=\frac{n(B-\alpha C)}{2 A}+2 n A=\frac{n^{2 / 3} E_{1}^{1 / 3}(4 k+5-5 \alpha k)}{2(k+1-\alpha k)^{2 / 3}}, \\
c=n^{2}\left[\frac{(B+C)}{2}+A^{2}-\frac{\alpha C}{2}\right]=\frac{3 n^{4 / 3} E_{1}^{2 / 3}(k+1-\alpha k)^{2 / 3}}{2}, \\
\Delta=b^{2}-4 a c=\frac{n^{4 / 3} E_{1}^{2 / 3}(4 k+5-5 \alpha k)^{2}}{4(k+1-\alpha k)^{4 / 3}}-6 n^{4 / 3} E_{1}^{2 / 3}(k+1-\alpha k)^{2 / 3} .
\end{array}\right.
$$

Therefore, it is easy to know that $\lambda_{1,2}$ are always a real number, $\lambda_{1}=\gamma_{1}=\lambda_{2}=\gamma_{2}$. If the discriminant $\Delta$ is greater than 0 , both characteristic roots are real number: $\lambda_{3}=\gamma_{3}$, $\lambda_{4}=\gamma_{4}$. From equations (77) and (78), we can know that when $\Delta>0$, the following expression holds:

$$
n^{4 / 3} E_{1}^{2 / 3}(4 k+5-5 \alpha k)^{2}>24 n^{4 / 3} E_{1}^{2 / 3}(k+1-\alpha k)^{2} .
$$

It is clear that

$$
|4 k+5-5 \alpha k|>|2 \sqrt{6}(k+1-\alpha k)| .
$$

When the discriminant $\Delta$ is less than 0 , conjugate complex roots exist, namely, $\lambda_{3,4}=\sigma_{3,4} \pm \mathrm{j} \omega_{3,4}$. From equations (77) and (78), we can know that when $\Delta<0$, the following expression holds:

$$
|4 k+5-5 \alpha k|<|2 \sqrt{6}(k+1-\alpha k)| .
$$

(4) Stability Analysis for Realization 2 ( $k$ Is Fixed and $\alpha$ Is Control Parameter)

(1) No equilibrium point exists in realization 2

If no equilibrium point exists in realization 2 , from equations (67) (68), it is known that the following expression is satisfied:

$$
k+1-\alpha k<0 .
$$

Therefore, from equation (82), when $k<-1$, it is clear that $0<\alpha<((k+1) / k)$ and when $-1<k<0$, we know that $\alpha$ does not exist, or when $0<k$, we know that $\alpha>((k+1) / k)$.

(2) A stable equilibrium point exists in realization 2

(2.1) Assuming there are all negative real characteristic roots

In this case, $\lambda_{1}, \lambda_{2}, \lambda_{3}$, and $\lambda_{4}$ are all less than 0 , and the system has a stable solution. We know that, owing to $k+1-\alpha k>0$, from equation (75), it is clear that $\lambda_{1}$ and $\lambda_{2}$ are always less than 0 . To make $\lambda_{3,4}<0$, according to Vieta theorem, we know that $\lambda_{3}+\lambda_{4}=-b$ and $\lambda_{3} \lambda_{4}=c$. From equation (78), it is known that always $c>0$, and owing to $k+1-\alpha k>0$, from equation (78), if $4 k+5-5 \alpha k>0$, we have $-b<0$, then $\lambda_{3,4}<0$. In this case, the absolute value sign in equation (80) can be removed; therefore, the following expression can be obtained:

$$
4 k+5-5 \alpha k>2 \sqrt{6}(k+1-\alpha k) .
$$

From expression (83), we can obtain the following expression:

$$
\alpha k(5-2 \sqrt{6})<k(4-2 \sqrt{6})+5-2 \sqrt{6} .
$$

In summary, if there are all negative real characteristic roots, the system is stable when the following inequalities are satisfied:

$$
\left\{\begin{array}{l}
\alpha k<k+1, \\
5 \alpha k<4 k+5 \\
\alpha k(5-2 \sqrt{6})<k(4-2 \sqrt{6})+5-2 \sqrt{6} .
\end{array}\right.
$$

(2.2) Assuming two real roots and two conjugate complex roots 
In this case, the four characteristic roots behave as $\lambda_{1}=\gamma_{1}=\lambda_{2}=\gamma_{2}$ and $\lambda_{3,4}=\sigma_{3,4} \pm j \omega_{3,4}$, where $\lambda_{1}$ and $\lambda_{2}$ are two negative real roots and $\lambda_{3}$ and $\lambda_{4}$ are two conjugate complex roots. When $\lambda_{1}, \lambda_{2}, \sigma_{3}$, and $\sigma_{4}$ are all less than 0 , the system has a stable solution.

From equation (77), we know $\sigma_{3,4}=-b / 2$. Owing to $\lambda_{1,2}<0$, to make $\sigma_{3,4}<0$, we have $-b<0$, from equation (78), we can obtain $4 k+5-5 \alpha k>0$.

In this case, the absolute value sign in expression (81) can be removed; therefore, the following expression (86) can be obtained:

$$
4 k+5-5 \alpha k<2 \sqrt{6}(k+1-\alpha k) .
$$

From expression (86), we can obtain that

$$
\alpha k(5-2 \sqrt{6})>k(4-.2 \sqrt{6})+5-2 \sqrt{6} .
$$

In summary, the system is stable when the following inequalities are satisfied:

$$
\left\{\begin{array}{l}
\alpha k<k+1 \\
5 \alpha k<4 k+5 \\
\alpha k(5-2 \sqrt{6})>k(4-.2 \sqrt{6})+5-2 \sqrt{6}
\end{array}\right.
$$

Combining expressions (85) and (88), a stable equilibrium point exists in realization 2 , and the following equations are satisfied:

$$
\left\{\begin{array}{l}
\alpha k<k+1 \\
5 \alpha k<4 k+5
\end{array}\right.
$$

Case 1: when $k>0$, expression (89) can be simplified to

$$
\left\{\begin{array}{l}
\alpha<\frac{k+1}{k}, \\
\alpha<\frac{4 k+5}{5 k} .
\end{array}\right.
$$

When $k>0$, it is easy to know that $((4 k+5) / 5 k)>0$ and $((k+1) / k)>0$, and it is clear that $((k+1) / k)-((4 k+$ $5) / 5 k)>0$ and $0<((4 k+5) / 5 k)<((k+1) / k)$. Therefore, from expression (90), when $k>0$, we know that $0<\alpha<((4 k+5) / 5 k)$.

Case 2: when $k<0$, expression (89) can be simplified to

$$
\left\{\begin{array}{l}
\alpha>\frac{k+1}{k} \\
\alpha>\frac{4 k+5}{5 k}
\end{array}\right.
$$

It is clear that $((k+1) / k)-((4 k+5) / 5 k)>0$ and $((4 k+5) / 5 k)<((k+1) / k)$. Owing to $\alpha>0$, when $-1<k<0$, it clear that $((k+1) / k)<0$. Therefore, from expression (91), when $-1<k<0$, we know that $\alpha>0$.

When $k<-1$, it clear that $((k+1) / k)>0$. Therefore, from expression (91), when $k<-1$, we know that $\alpha>((k+1) / k)$.

In conclusion, we can obtain following several cases: Case $1: k>0$ and $0<\alpha<((4 k+5) / 5 k)$

Case $2:-1<k<0$ and $\alpha>0$

Case 3: $k<-1$ and $\alpha>((k+1) / k)$

(3) An unstable equilibrium point exists in realization 1 (3.1)Assuming characteristic roots are all real and at least one is a positive

As long as one characteristic root is greater than 0 , the equilibrium point of the system is unstable. Owing to $k+1-\alpha k>0, \lambda_{1}$ and $\lambda_{2}$ are always less than 0 and only $\lambda_{3}$ or $\lambda_{4}$ are greater than 0 . According to Vieta theorem, it is known that $c>0$, and owing to $k+1-\alpha k>0$, from equation (78), if $4 k+5-5 \alpha k<0$, we have $-b>0$, then $\lambda_{3,4}>0$.

In this case, the number in the left absolute value sign of expression (80) is less than zero, right absolute value sign of expression (80) is greater than zero, and the absolute value signs are removed; expression (92) can be derived as follows:

$$
5 \alpha k-5-4 k>2 \sqrt{6}(k+1-\alpha k) .
$$

From expression (92), we can obtain

$$
\alpha k(5+2 \sqrt{6})>k(4+2 \sqrt{6})+5+2 \sqrt{6} .
$$

In summary, $\lambda_{1}$ and $\lambda_{2}$ are less than 0 and $\lambda_{3}$ and $\lambda_{4}$ greater than 0 . The system equilibrium point is the saddle point of index 2 , when the following inequalities are satisfied:

$$
\left\{\begin{array}{l}
\alpha k<k+1 \\
5 \alpha k>4 k+5 \\
\alpha k(5+2 \sqrt{6})>k(4+2 \sqrt{6})+5+2 \sqrt{6}
\end{array}\right.
$$

It is easy to know that for any $k,((k(4+2 \sqrt{6})+5+$ $2 \sqrt{6}) / k(5+2 \sqrt{6}))-((k+1) / k)<0$ and $((4 k+$ $5) / 5 k)-((k(4+2 \sqrt{6})+5+2 \sqrt{6}) / k(5+2 \sqrt{6}))<0$, so we have $((4 k+5) / 5 k)<((k(4+2 \sqrt{6})+5+$ $2 \sqrt{6}) / k(5+2 \sqrt{6}))<((k+1) / k)$. We discuss following several cases:

Case 1: when $k>0$, expression (94) is simplified as 


$$
\left\{\begin{array}{l}
\alpha<\frac{k+1}{k}, \\
\alpha>\frac{4 k+5}{5 k}, \\
\alpha>\frac{k(4+2 \sqrt{6})+5+2 \sqrt{6}}{k(5+2 \sqrt{6})} .
\end{array}\right.
$$

Therefore, when $k>0$, we have $((k(4+$ $2 . \sqrt{6})+5+2 \sqrt{6}) / k(5+2 \sqrt{6}))<\alpha<((1+k) / k)$.

Case 2: when $k<0$, expression (94) is simplified as

$$
\left\{\begin{array}{l}
\alpha>\frac{k+1}{k}, \\
\alpha<\frac{4 k+5}{5 k}, \\
\alpha<\frac{k(4+2 \sqrt{6})+5+2 \sqrt{6}}{k(5+2 \sqrt{6})} .
\end{array}\right.
$$

Therefore, when $k<0, \alpha$ does not exist.

In conclusion, we can obtain following several cases:

Case $1: \quad k>0$ and $((k(4+2 \sqrt{6})+5+2 \sqrt{6}) / k$ $(5+2 \sqrt{6}))<\alpha<((1+k) / k)$

Case 2: $k<0$ and $\alpha$ does not exist

If $k$ and $\alpha$ satisfy above relation, the system equilibrium point is the saddle point of index 2, which is unstable.

(3.2) Assuming two negative real roots and two conjugate complex roots

In this case, the four characteristic roots behave as $\lambda_{1}=\gamma_{1}=\lambda_{2}=\gamma_{2}$ and $\lambda_{3,4}=\sigma_{3,4} \pm j \omega_{3,4}$, where $\lambda_{1}$ and $\lambda_{2}$ are two negative real roots and $\lambda_{3}$ and $\lambda_{4}$ are two conjugate complex roots.

Owing to $\lambda_{1}$ and $\lambda_{2}$ are always less than 0 , when $\sigma_{3}$ and $\sigma_{4}$ are greater than 0 , the equilibrium point of the system is unstable and it is the saddle-focus point of index 2. From (77), we know $\sigma_{3,4}=-b / 2$. To make $\sigma_{3,4}>0$, we have $-b>0$. From equation (78), it is known that $4 \alpha-5+5 \alpha \mathrm{k}<0$.

In this case, the absolute value sign in expression (81) can be removed; therefore, the following expression (97) can be obtained:

$$
5 \alpha k-5-4 k<2 \sqrt{6}(k+1-\alpha k) .
$$

From expression (97), we can obtain that

$$
\alpha k(5+2 \sqrt{6})<k(4+2 \sqrt{6})+5+2 \sqrt{6} .
$$

In summary, $\lambda_{1}$ and $\lambda_{2}$ are less than 0 and $\sigma_{3}$ and $\sigma_{4}$ are greater than 0 ; the system equilibrium point is unstable and it is the saddle-focus point of index 2 when the following inequalities are satisfied:

$$
\left\{\begin{array}{l}
\alpha k<k+1, \\
5 \alpha k>4 k+5 \\
\alpha k(5+2 \sqrt{6})<k(4+2 \sqrt{6})+5+2 \sqrt{6} .
\end{array}\right.
$$

By referring the classification results of expression (94), we can obtain the following several cases:

Case $1: k>0$ and $((4 k+5) / 5 k)<\alpha<((k(4+2 \cdot \sqrt{6})+$ $5+2 \sqrt{6}) / k(5+2 \sqrt{6}))$

Case 2: $k<0$ and $\alpha$ does not exist

If $k$ and $\alpha$ satisfy above relation, the system equilibrium point is the saddle-focus point of index 2 , which is unstable.

In brief, for the scenario of the heat source in the left and right sides both are positive $(k>0)$, from the above discussion, we can form a criterion shown in Table 1 to determine the stability of the system shown in Figure 1 when $K$ is fixed, $\alpha$ is the control parameter, and $k>0$. According to the values of $\alpha$ in Table 1, we can know whether there is an equilibrium point and the equilibrium point is stable or not.

We compared Table 1 with the part $k>0$ of Table 1 in [35]. From these two tables, we can know that the results (whether the equilibrium point is stable or not) are roughly the same as those of [35]. However, our results are more specific and more detailed when the equilibrium points are unstable. In the unstable equilibrium point interval corresponding [35], we obtain two different subintervals corresponding to the saddle point of index 2 and the saddle focal equilibrium point of index 2 , respectively. This is because our model has four characteristic roots and only two in [35]. The brief description is as follows. The equilibrium point is unstable in realization 1 , when $\alpha$ is in the interval of $((1 /(1+k)),(5 /(5 k+4)))$, and this interval can be divided into two parts, namely, $\alpha \in((1 /(k+1)),((5+2 \sqrt{6}) /$ $(k(5+2 \sqrt{6})+4+2 \sqrt{6})))$, where the unstable equilibrium point is the saddle point of index 2 and $\alpha \in(((5+2 \sqrt{6}) /$ $(k(5+2 \sqrt{6})+4+2 \sqrt{6})),(5 /(5 k+4)))$, where the unstable equilibrium point is the saddle-focus point of index 2 . The equilibrium point is unstable in realization 2 , when $\alpha$ is in the interval of $(((4 k+5) / 5 k),((k+1) / k))$, and we divide this interval into two parts, namely, $\alpha \in(((4 k+5) / 5 k)$, $((k(4+2 \sqrt{6})+5+2 \sqrt{6}) / k(5+2 \sqrt{6})))$, where the unstable equilibrium point is the saddle point of index 2 and $\alpha \in(((k(4+2 \sqrt{6})+5+2 \sqrt{6}) /(k(5+2 \sqrt{6}))),((k+1) / k))$, where the unstable equilibrium point the saddle-focus point of index 2 . 
TABLE 1: Criterion for scenario 1 ( $(k$ is fixed, $\alpha$ is control parameter, and $k>0)$.

\begin{tabular}{cccc}
\hline$k$ & $\alpha$ & Realization 1 & Realization 2 \\
\hline & $((0,(1 /(k+1)))$ & No & Stable \\
& $(((5+2 \sqrt{6}) /(k(5+2 \sqrt{6})+4+2 \sqrt{6})),(5 /(5 k+4)))$ & Stable & Stable \\
$(0,+\infty)$ & $((5 /(5 k+4)),((4 k+5) / 5 k))$ & Saddle point of index 2 & Stable \\
& $(((4 k+5) / 5 k),((k(4+2 \sqrt{6})+5+2 \sqrt{6}) / k(5+2 \sqrt{6})))$ & Stable & Stable \\
& $(((k(4+2 \sqrt{6})+5+2 \sqrt{6}) / k(5+2 \sqrt{6})),((k+1) / k))$ & Stable & Saddle-focus point of index 2 \\
& $(((k+1) / k),+\infty)$ & Stable & Saddle point of index 2 \\
& & No
\end{tabular}

For the scenario of the heat source in the left is positive and the right is negative $(k<0)$, from the above discussion, we can form a criterion as shown in Table 2 to determine the stability of the system shown in Figure 1 when $K$ is fixed, $\alpha$ is the control parameter, and $k<0$. According to the values of $\alpha$ in Table 2, we can know whether there is an equilibrium point and the equilibrium point is stable or not.

We compared Table 2 with the part $k<0$ of Table 1 in [35]. From these two tables, we can know the results (whether the equilibrium point is stable or not) are roughly the same as those in [35]. However, similarly, our results are more specific and more detailed when the equilibrium points are unstable. In the unstable equilibrium interval corresponding to literature [35], we obtain two different subintervals corresponding to the saddle point of index 2 and the saddle focal equilibrium point of index 2 , respectively. This is because our model has four characteristic roots and only two in [35].

\subsubsection{Stability Analysis for Scenario 2 ( $\alpha$ Is Fixed and $k I s$ Control Parameter)}

(1) Stability Analysis for Realization 1 ( $\alpha$ Is Fixed and $k I s$ Control Parameter). In this scenario, the characteristic equation is the same as that of scenario 1.

(1) No equilibrium point exists in realization 1

In point (1) of Section 2.3.1, we already know that no equilibrium point exists in realization 1, when the following inequalities (equation (42) in Section 2.3.1) are satisfied:

$$
\alpha k-1+\alpha<0
$$

Therefore, from expression (98), when $\alpha>0$, it is clear that $k<((1-\alpha) / \alpha)$.

(2) A stable equilibrium point exists in realization 1 In point (2) of Section 2.3.1, we already know that the system was stable, when the following inequalities (expression (49) in Section 2.3.1) are satisfied:

$$
\left\{\begin{array}{l}
\alpha(k+1)-1>0, \\
\alpha(5 k+4)-5>0
\end{array}\right.
$$

Taking out the coefficient $k$ of expression (101), we can obtain expression (102):

$$
\left\{\begin{array}{l}
k \alpha>1-\alpha, \\
5 k \alpha>5-4 \alpha .
\end{array}\right.
$$

Owing to $\alpha>0$, expression (102) are simplified as

$$
\left\{\begin{array}{l}
k>\frac{1-\alpha}{\alpha}, \\
k>\frac{5-4 \alpha}{5 \alpha}
\end{array}\right.
$$

Owing to $\alpha>0$, it is clear that $((5-4 \alpha) / 5 \alpha)-((1-$ $\alpha) / \alpha)>0$ and $((5-4 \alpha) / 5 \alpha)>((1-\alpha) / \alpha)$; therefore, from expression (101), when $\alpha>0$, we know that $((5-4 \alpha) / 5 \alpha)<k$.

(3) An unstable equilibrium point exists in realization 1 (3.1) Assuming characteristic roots are all real and at least one is a positive

In point (3.1) of Section 2.3.1, we already know that the equilibrium point of the system is unstable and it is the saddle point of index 2, when the following inequalities (expression (55) in Section 2.3.1) are satisfied:

$$
\left\{\begin{array}{l}
\alpha k+\alpha-1>0 \\
5 \alpha k+4 \alpha-5<0 \\
\alpha[k(2 \sqrt{6}+5)+2 \sqrt{6}+4]<5+2 \sqrt{6}
\end{array}\right.
$$

Taking out the coefficient $k$ of expression (104), we can obtain the following expression:

$$
\left\{\begin{array}{l}
k \alpha>1-\alpha \\
5 k \alpha<5-4 \alpha \\
(5+2 \sqrt{6}) k \alpha<5+2 \sqrt{6}-\alpha(4+2 \sqrt{6}) .
\end{array}\right.
$$

Owing to $\alpha>0$, expression (105) is simplified: 
TABLE 2: Criterion for scenario 1 ( $k$ is fixed, $\alpha$ is control parameter and $k<0$ ).

\begin{tabular}{|c|c|c|c|}
\hline$k$ & $\alpha$ & Realization 1 & Realization 2 \\
\hline$(-(4 / 5), 0)$ & $\begin{array}{c}(0,(1 /(k+1))) \\
((1 /(k+1)),((2 \sqrt{6}+5) /(k(2 \sqrt{6}+5)+2 \sqrt{6}+4))) \\
(((2 \sqrt{6}+5) /(k(2 \sqrt{6}+5)+2 \sqrt{6}+4)),(5 /(5 k+4))) \\
((5 /(5 k+4)),+\infty)\end{array}$ & $\begin{array}{c}\text { No } \\
\text { Saddle point of index } 2 \\
\text { Saddle-focus point of index } 2 \\
\text { Stable }\end{array}$ & $\begin{array}{l}\text { Stable } \\
\text { Stable } \\
\text { Stable } \\
\text { Stable }\end{array}$ \\
\hline$(((-2 \sqrt{6}-4) /(2 \sqrt{6}+5)),-(4 / 5))$ & $\begin{array}{c}(0,(1 /(k+1))) \\
((1 /(k+1)),((5+2 \sqrt{6}) /(k(5+2 \sqrt{6})+4+2 \sqrt{6}))) \\
(((5+2 \sqrt{6}) /(k(5+2 \sqrt{6})+4+2 \sqrt{6})),+\infty)\end{array}$ & $\begin{array}{c}\text { No } \\
\text { Saddle point of index } 2 \\
\text { Saddle-focus point of index } 2\end{array}$ & $\begin{array}{l}\text { Stable } \\
\text { Stable } \\
\text { Stable }\end{array}$ \\
\hline$(-1,((-2 \sqrt{6}-4) /(2 \sqrt{6}+5)))$ & $\begin{array}{c}(0,(1 /(k+1))) \\
((1 /(k+1)),+\infty) \\
\end{array}$ & $\begin{array}{c}\text { No } \\
\text { Saddle point of index } 2 \\
\end{array}$ & $\begin{array}{l}\text { Stable } \\
\text { Stable }\end{array}$ \\
\hline$(-\infty,-1)$ & $\begin{array}{c}(0,((1+k) / k)) \\
(((1+k) / k),+\infty)\end{array}$ & $\begin{array}{l}\text { No } \\
\text { No }\end{array}$ & $\begin{array}{c}\text { No } \\
\text { Stable }\end{array}$ \\
\hline
\end{tabular}

$$
\left\{\begin{array}{l}
k>\frac{1-\alpha}{\alpha}, \\
k<\frac{5-4 \alpha}{5 \alpha}, \\
k<\frac{5+2 \sqrt{6}-\alpha(4+2 \sqrt{6})}{(5+2 \sqrt{6}) \alpha} .
\end{array}\right.
$$

It is easy to know that, for any $\alpha>0,((1-\alpha) / \alpha)-((5+$ $2 \sqrt{6}-\alpha(4+2 \sqrt{6})) /(\alpha(5+2 \sqrt{6})))<0$ and $\quad((5+$ $2 \sqrt{6}-\alpha(4+2 \sqrt{6})) / \alpha(5+2 \sqrt{6}))-((5-4 \alpha) / 5 \alpha)<0$, so we have $((1-\alpha) / \alpha)<((5+2 \sqrt{6}-\alpha(4+$ $2 \sqrt{6})) / \alpha(5+2 \sqrt{6}))<((5-4 \alpha) / 5 \alpha)$. Therefore, from expression (106), it can be known that, when $\alpha>0$, we know that $((1-\alpha) / \alpha)<k<((5+2 \sqrt{6}-\alpha(4+$ $2 \sqrt{6})) / \alpha(5+2 \sqrt{6}))$.

If $\alpha$ and $k$ satisfy above relation, the system equilibrium point is the saddle point of index 2, which is unstable.

(3.2) Assuming two negative real roots and two conjugate complex roots

In point (3.2) of Section 2.3.1, we already know that the equilibrium point of the system is unstable and it is the saddle-focus point of index 2 , when the following inequalities (expression (62) in Section 2.3.1) are satisfied:

$$
\left\{\begin{array}{l}
\alpha(k+1)-1>0 \\
\alpha(5 k+4)-5<0 \\
\alpha[k(5+2 \sqrt{6})+4+2 \sqrt{6}]>2 \sqrt{6}+5
\end{array}\right.
$$

Taking out the coefficient $k$ of expression (107), we can obtain the following expression:

$$
\left\{\begin{array}{l}
k \alpha>1-\alpha, \\
5 k \alpha<5-4 \alpha \\
(5+2 \sqrt{6}) k \alpha>5+2 \sqrt{6}-\alpha(4+2 \sqrt{6}) .
\end{array}\right.
$$

Owing to $\alpha>0$, expression (108) is simplified:

$$
\left\{\begin{array}{l}
k>\frac{1-\alpha}{\alpha}, \\
k<\frac{5-4 \alpha}{5 \alpha}, \\
k>\frac{5+2 \sqrt{6}-\alpha(4+2 \sqrt{6})}{(5+2 \sqrt{6}) \alpha} .
\end{array}\right.
$$

By referring the solution procedure of expression (104), we can obtain the following result. When $\alpha>0$, we know that $((5+2 \sqrt{6}-\alpha(4+2 \sqrt{6})) / \alpha(5+2 \sqrt{6}))<$ $k<((5-4 \alpha) / 5 \alpha)$.

If $\alpha$ and $k$ satisfy the above relation, the system equilibrium point is the saddle-focus point of index 2, which is unstable.

(2) Stability Analysis for Realization 2 ( $\alpha$ Is Fixed, $k$ Is Control Parameter)

(1) No equilibrium point exists in realization 2

In point (1) of (4) of Section 2.3.1, we already know that no equilibrium point exists in realization 2 , when the following inequalities (expression (82) in Section 2.3.1) are satisfied:

$$
k+1-\alpha k<0 \text {. }
$$

Therefore, from expression (110), when $0<\alpha<1$, it is clear that $k<(1 /(\alpha-1))$ and when $\alpha>1$, we know that $k>(1 /(\alpha-1))$.

(2) A stable equilibrium point exists in realization 2 In point (2) of (4) of Section 2.3.1, we already know that the system was stable, when the following inequalities (expression (89) in (4) of Section 2.3.1) are satisfied:

$$
\left\{\begin{array}{l}
\alpha k<k+1 \\
5 \alpha k<4 k+5
\end{array}\right.
$$

Taking out the coefficient $k$ of expression (111), we can obtain the following expression: 


$$
\left\{\begin{array}{l}
k(\alpha-1)<1, \\
k(5 \alpha-4)<5
\end{array}\right.
$$

Case 1: when $\alpha>1$, it is easy to know that $\alpha-1>0$ and $5 \alpha-4>0$, and then expression (112) can be simplified to

$$
\left\{\begin{array}{l}
k<\frac{1}{\alpha-1}, \\
k<\frac{5}{5 \alpha-4} .
\end{array}\right.
$$

From $\alpha-1>0$ and $5 \alpha-4>0$, it is clear that $(5 /(5 \alpha-4))-(1 /(\alpha-1))<0 \quad$ and $0<(5 /(5 \alpha-$ $4))<(1 /(\alpha-1))$. Therefore, from expression (113), when $\alpha>1$, we know that $k<(-5 /(4-5 \alpha))$.

Case 2: when $(4 / 5)<\alpha<1$, it is easy to know that $\alpha-1<0$ and $5 \alpha-4>0$; then expression (112) can be simplified to

$$
\left\{\begin{array}{l}
k>\frac{1}{\alpha-1}, \\
k<\frac{5}{5 \alpha-4} .
\end{array}\right.
$$

From $\alpha-1<0$ and $5 \alpha-4>0$, it is clear that $(5 /(5 \alpha-4))-(1 /(\alpha-1))>0, \quad(1 /(\alpha-1))<0 \quad$ and $0<(5 /(5 \alpha-4))$. Therefore, from expression (112), when $(4 / 5)<\alpha<1$, we know that $(1 /(\alpha-1))<$ $k<(5 /(5 \alpha-4))$.

Case 3: when $0<\alpha<(4 / 5)$, it is easy to know that $\alpha-1<0$ and $5 \alpha-4<0$, then expression (112) can be simplified to

$$
\left\{\begin{array}{l}
k>\frac{1}{\alpha-1} \\
k>\frac{5}{5 \alpha-4} .
\end{array}\right.
$$

From $\alpha-1<0$ and $5 \alpha-4<0$, it is clear that $(5 /(5 \alpha-4))-(1 /(\alpha-1))<0$ and $(5 /(5 \alpha-4))<$ $(1 /(\alpha-1))<0$, Therefore, from expression (115), when $0<\alpha<(4 / 5)$, we know that $(1 /(\alpha-1))<k$. In conclusion, we can obtain following several cases:

Case 1: $\alpha>1$ and $k<(-5 /(4-5 \alpha))$

Case 2: $\quad(4 / 5)<\alpha<1 \quad$ and $\quad(1 /(\alpha-1))<k<$
$(5 /(5 \alpha-4))$

Case 3: $0<\alpha<(4 / 5)$ and $(1 /(\alpha-1))<k$

(3) An unstable equilibrium point exists in realization 2
(3.1) Assuming characteristic roots are all real and at least one is a positive

In point (3.1) of Section 2.3.1, we already know that the equilibrium point of the system is unstable and it is the saddle point of index 2, when the following inequalities (expression (94) in Section 2.3.1) are satisfied:

$$
\left\{\begin{array}{l}
\alpha k<k+1, \\
5 \alpha k>4 k+5 \\
\alpha k(5+2 \sqrt{6})>k(4+2 \sqrt{6})+5+2 \sqrt{6} .
\end{array}\right.
$$

Taking out the coefficient $k$ of expression (116), we can obtain the following expression:

$$
\left\{\begin{array}{l}
k(1-\alpha)+1>0, \\
k(4-5 \alpha)+5<0, \\
k[\alpha(5+2 \sqrt{6})+(-2 \sqrt{6}-4)]>5+2 \sqrt{6}
\end{array}\right.
$$

Firstly, we consider the coefficient of $k$ on the left side of third inequality in expression (117) is greater than 0 , namely, $\alpha(5+2 \sqrt{6})+(-2 \sqrt{6}-4)>0$, and we can obtain $\alpha>((4+2 \sqrt{6}) /(5+2 \sqrt{6}))$. Then, we consider the coefficient of $\alpha$ on the left side of third inequality in (117) is less than 0, namely, $\alpha(5+2 \sqrt{6})+$ $(-2 \sqrt{6}-4)<0$, and we can obtain $\alpha<((4+$ $2 \sqrt{6}) /(5+2 \sqrt{6}))$. It is easy to know that $0<(4 / 5)<((4+2 \sqrt{6}) /(5+2 \sqrt{6}))<1$. We discuss following several cases:

Case 1: when $((4+2 \sqrt{6}) /(5+2 \sqrt{6}))<\alpha<1$, owing to $0<(4 / 5)<((4+2 \sqrt{6}) /(5+2 \sqrt{6}))<1$, it is easy to know that $4-5 \alpha<0, \quad 1-\alpha>0, \quad$ and $\alpha(5+$ $2 \sqrt{6})+(-2 \sqrt{6}-4)>0$; equation (117) is simplified as

$$
\left\{\begin{array}{l}
k>\frac{-1}{1-\alpha}, \\
k>\frac{-5}{4-5 \alpha}, \\
k>\frac{5+2 \sqrt{6}}{\alpha(5+2 \sqrt{6})-4-2 \sqrt{6}} .
\end{array}\right.
$$

From $4-5 \alpha<0,1-\alpha>0$, and $\alpha(5+2 \sqrt{6})+(-2 \sqrt{6}$ $-4)>0$, it is clear that $(-1 /(1-\alpha))-(-5 /(4-5 \alpha))<0$, $(-5 /(4-5 \alpha))-((5+2 \sqrt{6}) /(\alpha(5+2 \sqrt{6})-4-2 \sqrt{6}))$ $<0$, and $(-1 /(1-\alpha))<(-5 /(4-5 \alpha))<((5+2 \sqrt{6}) /$ $(\alpha(5+2 \sqrt{6})-4-2 \sqrt{6}))$. Therefore, when $((4+2$ $\sqrt{6}) /(5+2 \sqrt{6}))<\alpha<1$, we know that $k>((5+2 \sqrt{6}) /$ $(\alpha(5+2 \sqrt{6})-4-2 \sqrt{6}))$.

Case 2: when $\alpha>1$, owing to $0<(4 / 5)<((4+2 \sqrt{6}) /$ $(5+2 \sqrt{6}))<1$, it is easy to know that $4-5 \alpha<0$, 
$1-\alpha<0$, and $\alpha(5+2 \sqrt{6})+(-2 \sqrt{6}-4)>0$; expression (117) is simplified as

$$
\left\{\begin{array}{l}
k<\frac{-1}{1-\alpha}, \\
k>\frac{-5}{4-5 \alpha}, \\
k>\frac{5+2 \sqrt{6}}{\alpha(5+2 \sqrt{6})-4-2 \sqrt{6}} .
\end{array}\right.
$$

From $4-5 \alpha<0,1-\alpha<0$, and $\alpha(5+2 \sqrt{6})+(-2 \sqrt{6}$ $-4)>0$, it is clear that $(-5 /(4-5 \alpha))-((5+2 \sqrt{6}) /(\alpha$ $(5+2 \sqrt{6})-4-2 \sqrt{6}))<0, \quad((5+2 \sqrt{6}) /(\alpha(5+2 \sqrt{6})$ $-4-2 \sqrt{6}))-(-1 /(1-\alpha))<0$, and $(-5 /(4-5 \alpha))<$ $((5+2 \sqrt{6}) /(\alpha(5+2 \sqrt{6})-4-2 \sqrt{6}))<(-1 /(1-\alpha))$. Therefore, when $\alpha>1$, we know that $((5+2 \sqrt{6}) /$ $(\alpha(5+2 \sqrt{6})-4-2 \sqrt{6}))<k<(-1 /(1-\alpha))$.

Case 3: when $(4 / 5)<\alpha<((4+2 \sqrt{6}) /(5+2 \sqrt{6}))$, owing to $0<(4 / 5)<((4+2 \sqrt{6}) /(5+2 \sqrt{6}))<1$, it is easy to know that $4-5 \alpha<0, \quad 1-\alpha>0$, and $\alpha(5+2 \sqrt{6})+(-2 \sqrt{6}-4)<0$; expression (117) are simplified as

$$
\left\{\begin{array}{l}
k>\frac{-1}{1-\alpha}, \\
k>\frac{-5}{4-5 \alpha}, \\
k<\frac{5+2 \sqrt{6}}{\alpha(5+2 \sqrt{6})-4-2 \sqrt{6}} .
\end{array}\right.
$$

From $\quad 4-5 \alpha<0, \quad 1-\alpha>0, \quad$ and $\quad \alpha(5+2 \sqrt{6})+$ $(-2 \sqrt{6}-4)<0$, it is clear that $((5+2 \sqrt{6}) /$ $(\alpha(5+2 \sqrt{6})-4-2 \sqrt{6}))-(-1 /(1-\alpha))<0,(-1 /(1-$ $\alpha))-(-5 /(4-5 \alpha))<0 \quad 0<(-5 /(4-5 \alpha))$ and $((5+2 \sqrt{6}) /(\alpha(5+2 \sqrt{6})-4-2 \sqrt{6}))<(-1 /(1-\alpha))$ $<0$. Therefore, when $(4 / 5)<\alpha<((4+2 \sqrt{6}) /$ $(5+2 \sqrt{6}))$, we know that $k$ does not exist.

Case 4: when $0<\alpha<(4 / 5)$, owing to $0<(4 / 5)<$ $((4+2 \sqrt{6}) /(5+2 \sqrt{6}))<1$, it is easy to know that $4-5 \alpha>0,1-\alpha>0$, and $\alpha(5+2 \sqrt{6})+(-2 \sqrt{6}-4)<0$; expression (117) is simplified as

$$
\left\{\begin{array}{l}
k>\frac{-1}{1-\alpha}, \\
k<\frac{-5}{4-5 \alpha}, \\
k<\frac{5+2 \sqrt{6}}{\alpha(5+2 \sqrt{6})-4-2 \sqrt{6}} .
\end{array}\right.
$$

From $4-5 \alpha>0,1-\alpha>0$, and $\alpha(5+2 \sqrt{6})+(-2 \sqrt{6}$ $-4)<0$, it is clear that $(-5 /(4-5 \alpha))-((5+2 \sqrt{6}) /$ $(\alpha(5+2 \sqrt{6})-4-2 \sqrt{6}))<0, \quad((5+2 \sqrt{6}) /(\alpha(5+2$ $\sqrt{6})-4-2 \sqrt{6}))-(-1 /(1-\alpha))<0$, and $(-5 /(4-$
$5 \alpha))<((5+2 \sqrt{6}) /(\alpha(5+2 \sqrt{6})-4-2 \sqrt{6}))<(-1 /(1$ $-\alpha))<0$. Therefore, when $0<\alpha<(4 / 5)$, we know that $k$ does not exist.

From what has been discussed above, we can obtain following relations between $\alpha$ and $k$.

Case 1: $((4+2 \sqrt{6}) /(5+2 \sqrt{6}))<\alpha<1$ and $k>((5+$ $2 \sqrt{6}) /(\alpha(5+2 \sqrt{6})-4-2 \sqrt{6}))$

Case 2: $\alpha>1$ and $((5+2 \sqrt{6}) /(\alpha(5+2 \sqrt{6})-4-$ $2 \sqrt{6}))<k<(-1 /(1-\alpha))$

Case 3: $0<\alpha<(4 / 5)$ and $k$ does not exist.

Case 4: $(4 / 5)<\alpha<((4+2 \sqrt{6}) /(5+2 \sqrt{6}))$ and $k$ does not exist.

If $\alpha$ and $k$ satisfy above relation, the system equilibrium point is the saddle point of index 2, which is unstable.

(3.2) Assuming two negative real roots and two conjugate complex roots

In point (3.2) of Section 2.3.1, we already know that the equilibrium point of the system is unstable and it is the saddle-focus point of index 2, when the following inequalities (expression (96) in Section 2.3.1) are satisfied:

$$
\left\{\begin{array}{l}
\alpha k<k+1, \\
5 \alpha k>4 k+5 \\
\alpha k(5+2 \sqrt{6})<k(4+2 \sqrt{6})+5+2 \sqrt{6} .
\end{array}\right.
$$

Taking out the coefficient $k$ of expression (122), we can obtain the following expression:

$$
\left\{\begin{array}{l}
k(1-\alpha)+1>0, \\
k(4-5 \alpha)+5<0, \\
k[\alpha(5+2 \sqrt{6})+(-2 \sqrt{6}-4)]<5+2 \sqrt{6} .
\end{array}\right.
$$

By referring the classification results of expression (117), we can obtain the following several cases:

Case $1:((4+2 \sqrt{6}) /(5+2 \sqrt{6}))<\alpha<1$ and $(-5 /(4-$ $5 \alpha))<k<((5+2 \sqrt{6}) /(\alpha(5+2 \sqrt{6})-4-2 \sqrt{6}))$

Case 2: $\quad \alpha>1$ and $(-5 /(4-5 \alpha))<k<\quad((5+2$ $\sqrt{6}) /(\alpha(5+2 \sqrt{6})-4-2 \sqrt{6}))$

Case 3: $0<\alpha<(4 / 5)$ and $k$ does not exist

Case 4: $\quad(4 / 5)<\alpha<((4+2 \sqrt{6}) /(5+2 \sqrt{6})) \quad$ and $k>(-5 /(4-5 \alpha))$

If $k$ and $\alpha$ satisfy above relation, the system equilibrium point is the saddle-focus point of index 2, which is unstable.

In brief, for the scenario $2, \alpha$ can be discussed in the following sections: $0<\alpha<(4 / 5), \quad(4 / 5)<\alpha<$ $((4+2 \sqrt{6}) /(5+2 \sqrt{6}))$, and $((4+2 \sqrt{6}) /(5+2 \sqrt{6}))$ $<\alpha<1$ and $1<\alpha$.

From the above discussion, we can form a criterion shown in Table 3 to determine the stability of the system shown in Figure 1; when $\alpha$ is fixed, $k$ is the control parameter. According to the values of $k$ in Table 3, we can know 
TABLE 3: Criterion for scenario 2 ( $\alpha$ is fixed and $k$ is control parameter).

\begin{tabular}{|c|c|c|c|}
\hline$\alpha$ & $k$ & Realization 1 & Realization 2 \\
\hline \multirow{4}{*}{$(0,(4 / 5))$} & $\begin{array}{c}(-\infty,(-1 /(1-\alpha))) \\
((-1 /(1-\alpha)),((5-2 \sqrt{6}) /(\alpha(5-2 \sqrt{6})+(2 \sqrt{6}-4)))) \\
(((5-2 \sqrt{6}) /(\alpha(5-2 \sqrt{6})+(2 \sqrt{6}-4))),((1-\alpha) / \alpha))\end{array}$ & $\begin{array}{l}\text { No } \\
\text { No } \\
\text { No }\end{array}$ & $\begin{array}{l}\text { No } \\
\text { Stable } \\
\text { Stable }\end{array}$ \\
\hline & $(((1-\alpha) / \alpha),((5+2 \sqrt{6}-\alpha(4+2 \sqrt{6})) / \alpha(5+2 \sqrt{6})))$ & $\begin{array}{l}\text { Saddle point of index } \\
2\end{array}$ & Stable \\
\hline & $(((5+2 \sqrt{6}-\alpha(4+2 \sqrt{6})) / \alpha(5+2 \sqrt{6})),((5-4 \alpha) / 5 \alpha))$ & $\begin{array}{l}\text { Saddle-focus point of } \\
\text { index } 2\end{array}$ & Stable \\
\hline & $(((5-4 \alpha) / 5 \alpha),+\infty)$ & Stable & Stable \\
\hline \multirow{5}{*}{$((4 / 5),((4+2 \sqrt{6}) /(5+2 \sqrt{6})))$} & $\begin{array}{c}(-\infty,(-1 /(1-\alpha))) \\
((-1 /(1-\alpha)),((1-\alpha) / \alpha))\end{array}$ & $\begin{array}{l}\text { No } \\
\text { No }\end{array}$ & $\begin{array}{c}\text { No } \\
\text { Stable }\end{array}$ \\
\hline & $(((1-\alpha) / \alpha),((5+2 \sqrt{6}-\alpha(4+2 \sqrt{6})) / \alpha(5+2 \sqrt{6})))$ & Saddle point of index & Stable \\
\hline & $(((5+2 \sqrt{6}-\alpha(4+2 \sqrt{6})) / \alpha(5+2 \sqrt{6})),((5-4 \alpha) / 5 \alpha))$ & $\begin{array}{l}\text { Saddle-focus point of } \\
\text { index } 2\end{array}$ & Stable \\
\hline & $(((5-4 \alpha) / 5 \alpha),((4-5 \alpha) /-5))$ & Stable & Stable \\
\hline & $((-5 /(4-5 \alpha)),+\infty)$ & Stable & $\begin{array}{l}\text { Saddle-focus point of } \\
\text { index } 2\end{array}$ \\
\hline \multirow{6}{*}{$(((4+2 \sqrt{6}) /(5+2 \sqrt{6})), 1)$} & $\begin{array}{c}(-\infty,(-1 /(1-\alpha))) \\
((-1 /(1-\alpha)),((1-\alpha) / \alpha))\end{array}$ & No & $\begin{array}{c}\text { No } \\
\text { Stable }\end{array}$ \\
\hline & $(((1-\alpha) / \alpha),((5+2 \sqrt{6}-\alpha(4+2 \sqrt{6})) / \alpha(5+2 \sqrt{6})))$ & Saddle point of index & Stable \\
\hline & $(((5+2 \sqrt{6}-\alpha(4+2 \sqrt{6})) / \alpha(5+2 \sqrt{6})),((5-4 \alpha) / 5 \alpha))$ & $\begin{array}{l}\text { Saddle-focus point of } \\
\text { index } 2\end{array}$ & Stable \\
\hline & $(((5-4 \alpha) / 5 \alpha),(-5 /(4-5 \alpha)))$ & Stable & Stable \\
\hline & $((-5 /(4-5 \alpha)),((5+2 \sqrt{6}) /(\alpha(5+2 \sqrt{6})-4-2 \sqrt{6})))$ & Stable & $\begin{array}{l}\text { Saddle-focus point of } \\
\text { index } 2\end{array}$ \\
\hline & $(((5+2 \sqrt{6}) /(\alpha(5+2 \sqrt{6})-4-2 \sqrt{6})),+\infty)$ & Stable & $\begin{array}{l}\text { Saddle point of index } \\
2\end{array}$ \\
\hline \multirow{7}{*}{$(1,+\infty)$} & $(-\infty,((1-\alpha) / \alpha))$ & No & Stable \\
\hline & $(((1-\alpha) / \alpha),((5+2 \sqrt{6}-\alpha(4+2 \sqrt{6})) / \alpha(5+2 \sqrt{6})))$ & $\begin{array}{l}\text { Saddle point of index } \\
2\end{array}$ & Stable \\
\hline & $(((5+2 \sqrt{6}-\alpha(4+2 \sqrt{6})) / \alpha(5+2 \sqrt{6})),((5-4 \alpha) / 5 \alpha))$ & $\begin{array}{l}\text { Saddle-focus point of } \\
\text { index } 2\end{array}$ & Stable \\
\hline & $(((5-4 \alpha) / 5 \alpha),(-5 /(4-5 \alpha)))$ & Stable & Stable \\
\hline & $((-5 /(4-5 \alpha)),((5+2 \sqrt{6}) /(\alpha(5+2 \sqrt{6})-4-2 \sqrt{6})))$ & Stable & $\begin{array}{c}\text { Saddle-focus point of } \\
\text { index } 2\end{array}$ \\
\hline & $(((5+2 \sqrt{6}) /(\alpha(5+2 \sqrt{6})-4-2 \sqrt{6})),(-1 /(1-\alpha)))$ & Stable & Saddle point of index \\
\hline & $((-1 /(1-\alpha)),+\infty)$ & Stable & No \\
\hline
\end{tabular}

whether there is an equilibrium point and the equilibrium point is stable or not.

We compared Table 3 with Table 2 in [35]. From these two tables, we can know the results (whether the equilibrium point is stable or not) are roughly the same as those in [35]. However, our results are more specific and more detailed when the equilibrium points are unstable. In the unstable equilibrium point interval corresponding to [35], we can obtain two different subintervals corresponding to the saddle point of index 2 and the saddle focal equilibrium point of index 2, respectively. This is because our model has four characteristic roots and only two in [35].

2.3.3. Stability Analysis for Scenario 3 (One Heat Sink and One Heat Source). For realization 1, the heat at the bottom releases to the right side. Therefore, we can obtain following equations:

$$
\begin{aligned}
& f_{1}=\frac{\mathrm{d} \Delta T_{1}}{\mathrm{~d} \tau}=-n \sqrt{\alpha \Delta T_{2}-\Delta T_{1}}\left(\Delta T_{1}-\Delta T_{3}\right), \\
& f_{2}=\frac{\mathrm{d} \Delta T_{2}}{\mathrm{~d} \tau}=-n \sqrt{\alpha \Delta T_{2}-\Delta T_{1}}\left(\Delta T_{2}-\Delta T_{1}\right)+E_{1}, \\
& f_{3}=\frac{\mathrm{d} \Delta T_{3}}{\mathrm{~d} \tau}=-\xi n \sqrt{\alpha \Delta T_{2}-\Delta T_{1}} \Delta T_{3}, \\
& f_{4}=\frac{\mathrm{d} \Delta T_{4}}{\mathrm{~d} \tau}=-\xi n \sqrt{\alpha \Delta T_{2}-\Delta T_{1}}\left(\Delta T_{4}-\Delta T_{2}\right) .
\end{aligned}
$$

For realization 2 , the heat at the bottom releases to zone 1. Therefore,

$$
\begin{aligned}
& f_{1}=\frac{\mathrm{d} \Delta T_{1}}{\mathrm{~d} \tau}=-n \sqrt{\Delta T_{1}-\alpha \Delta T_{2}}\left(\Delta T_{1}-\Delta T_{2}\right)+E_{1}, \\
& f_{2}=\frac{\mathrm{d} \Delta T_{2}}{\mathrm{~d} \tau}=-n \sqrt{\Delta T_{1}-\alpha \Delta T_{2}}\left(\Delta T_{2}-\Delta T_{4}\right),
\end{aligned}
$$




$$
\begin{aligned}
& f_{3}=\frac{\mathrm{d} \Delta T_{3}}{\mathrm{~d} \tau}=-\xi n \sqrt{\Delta T_{1}-\alpha \Delta T_{2}}\left(\Delta T_{3}-\Delta T_{1}\right), \\
& f_{4}=\frac{\mathrm{d} \Delta T_{4}}{\mathrm{~d} \tau}=-\xi n \sqrt{\Delta T_{1}-\alpha \Delta T_{2}} \Delta T_{4} .
\end{aligned}
$$

(1) Stability Analysis for Realization 1. The steady state solution (equilibrium points) of realization 1 with equations (124) (127) is denoted as $\left(\Delta T_{01}, \Delta T_{02}, \Delta T_{03}, \Delta T_{04}\right)$; in order to solve the equilibrium points, we can make that the right sides of equations (124) (127) equal to zero; the following equations hold:

$$
\begin{array}{r}
-n \sqrt{\alpha \Delta T_{2}-\Delta T_{1}}\left(\Delta T_{1}-\Delta T_{3}\right)=0, \\
-n \sqrt{\alpha \Delta T_{2}-\Delta T_{1}}\left(\Delta T_{2}-\Delta T_{1}\right)+E_{1}=0, \\
-\xi n \sqrt{\alpha \Delta T_{2}-\Delta T_{1}} \Delta T_{3}=0, \\
-\xi n \sqrt{\alpha \Delta T_{2}-\Delta T_{1}}\left(\Delta T_{4}-\Delta T_{2}\right)=0 .
\end{array}
$$

By solving equations (132) (135), we can obtain the value of equilibrium points as follows:

$$
\begin{aligned}
& \Delta T_{01}=\Delta T_{03}=0, \\
& \Delta T_{02}=\Delta T_{04}=\frac{E_{1}^{2 / 3}}{n^{2 / 3} \alpha^{1 / 3}} .
\end{aligned}
$$

According to the characteristic determinant equal to 0 , the solution of the differential equation can be discussed. The characteristic determinant equation is that

$$
|J(Q)-\lambda E|=\left|\begin{array}{cccc}
\frac{\partial f_{1}}{\partial \Delta T_{1}}-\lambda & \frac{\partial f_{1}}{\partial \Delta T_{2}} & \frac{\partial f_{1}}{\partial \Delta T_{3}} & \frac{\partial f_{1}}{\partial \Delta T_{4}} \\
\frac{\partial f_{2}}{\partial \Delta T_{1}} & \frac{\partial f_{2}}{\partial \Delta T_{2}}-\lambda & \frac{\partial f_{2}}{\partial \Delta T_{3}} & \frac{\partial f_{2}}{\partial \Delta T_{4}} \\
\frac{\partial f_{3}}{\partial \Delta T_{1}} & \frac{\partial f_{3}}{\partial \Delta T_{2}} & \frac{\partial f_{3}}{\partial \Delta T_{3}}-\lambda & \frac{\partial f_{3}}{\partial \Delta T_{4}} \\
\frac{\partial f_{4}}{\partial \Delta T_{1}} & \frac{\partial f_{4}}{\partial \Delta T_{2}} & \frac{\partial f_{4}}{\partial \Delta T_{3}} & \frac{\partial f_{4}}{\partial \Delta T_{4}}-\lambda
\end{array}\right|=0 .
$$

Combining equations (124) (127), we can obtain the following expression:

$$
\begin{aligned}
& \left|\begin{array}{cccc}
\frac{n\left(\Delta T_{01}-\Delta T_{03}\right)}{2 A}-n A-\lambda & -\frac{n \alpha\left(\Delta T_{01}-\Delta T_{03}\right)}{2 A} & n A & 0 \\
\frac{n\left(\Delta T_{02}-\Delta T_{01}\right)}{2 A}+n A & -\frac{n \alpha\left(\Delta T_{02}-\Delta T_{01}\right)}{2 A}-n A-\lambda & 0 & 0 \\
\frac{\xi n \Delta T_{03}}{2 A} & -\frac{\xi n \alpha \Delta T_{03}}{2 A} & -\xi n A-\lambda & 0 \\
\frac{\xi n\left(\Delta T_{04}-\Delta T_{02}\right)}{2 A} & -\frac{\xi n \alpha\left(\Delta T_{04}-\Delta T_{02}\right)}{2 A}+\xi n A & 0 & -\xi n A-\lambda
\end{array}\right|=0 \text {, } \\
& A=\sqrt{\alpha \Delta T_{02}-\Delta T_{01}}=\frac{\alpha^{1 / 3} E_{1}^{1 / 3}}{n^{1 / 3}}, \\
& B=\Delta T_{01}-\Delta T_{03}=0, \\
& C=\Delta T_{02}-\Delta T_{01}=\frac{E_{1}^{2 / 3}}{n^{2 / 3} \alpha^{1 / 3}} .
\end{aligned}
$$

Two other eigenvalues can be obtained according to the quadratic formula:
Combining equations (139) (142), we can obtain characteristic root as follows:

$$
\lambda_{1}=\lambda_{2}=-\xi n A=-\xi n \sqrt{\alpha \Delta T_{02}-\Delta T_{01}}=-\xi n^{2 / 3} E_{1}^{1 / 3} \alpha^{1 / 3} .
$$

$$
\lambda_{3,4}=\frac{-b \pm \sqrt{b^{2}-4 a c}}{2 a}=\frac{-(n \alpha C / 2 A)-2 n A \pm \sqrt{[(n \alpha C / 2 A)+2 n A]^{2}-4 n^{2}\left[(\alpha C / 2)+A^{2}\right]}}{2}
$$


where

$$
\left\{\begin{array}{l}
a=1, \\
b=\frac{n \alpha C}{2 A}+2 n A=\frac{3 n^{2 / 3} E_{1}^{1 / 3} \alpha^{1 / 3}}{2}, \\
c=n^{2}\left[\frac{\alpha C}{2}+A^{2}\right]=\frac{3 E_{1}^{2 / 3} \alpha^{2 / 3} n^{4 / 3}}{2}, \\
\Delta=b^{2}-4 a c=\frac{9 n^{4 / 3} E_{1}^{2 / 3} \alpha^{2 / 3}}{4}-6 E_{1}^{2 / 3} \alpha^{2 / 3} n^{4 / 3}=-\frac{15 n^{4 / 3} E_{1}^{2 / 3} \alpha^{2 / 3}}{4} .
\end{array}\right.
$$

It is easy to know that $\lambda_{1,2}$ are always real number $\lambda_{1}=\gamma_{1}=\lambda_{2}=\gamma_{2}$. Owing to $\alpha>0, E_{1}>0$, and $n>0$, from equation (143), it is clear that $\lambda_{1,2}$ are always less than 0 . From equation (145), we know that $\Delta<0$. Since the discriminant $\Delta<0$, we know that $\lambda_{3}$ and $\lambda_{4}$ are always conjugate complex numbers $\lambda_{3,4}=\sigma_{3,4} \pm j \omega_{3,4}$. Hence, the stability related with $\lambda_{3,4}$ is determined by the real part $\sigma_{3,4}$. Owing to $\alpha>0, E_{1}>0, n>0$, from equations (144) and (145), we know that $\sigma_{3,4}=-b<0$. As a result, $\lambda_{1}, \lambda_{2}$, and $\sigma_{3,4}$ are negative. It is clear that the equilibrium point is stable in realization 1.

(2) Stability Analysis for Realization 2. The steady state solution (equilibrium points) of realization 2 with equations (127) (130) is denoted as $\left(\Delta T_{01}, \Delta T_{02}, \Delta T_{03}, \Delta T_{04}\right)$. In order to solve the equilibrium points, we can make that the right sides of equations (127) (130) equal to zero; the following equations hold:

$$
\begin{aligned}
-n \sqrt{\Delta T_{1}-\alpha \Delta T_{2}}\left(\Delta T_{1}-\Delta T_{2}\right)+E_{1} & =0, \\
-n \sqrt{\Delta T_{1}-\alpha \Delta T_{2}}\left(\Delta T_{2}-\Delta T_{4}\right) & =0, \\
-\xi n \sqrt{\Delta T_{1}-\alpha \Delta T_{2}}\left(\Delta T_{3}-\Delta T_{1}\right) & =0, \\
-\xi n \sqrt{\Delta T_{1}-\alpha \Delta T_{2}} \Delta T_{4} & =0 .
\end{aligned}
$$

By solving equations (146) (149), we can obtain the value of equilibrium point as follows:

$$
\begin{aligned}
& \Delta T_{01}=\Delta T_{03}=\frac{E_{1}^{2 / 3}}{n^{2 / 3}}, \\
& \Delta T_{02}=\Delta T_{04}=0 .
\end{aligned}
$$

According to the characteristic determinant equal to 0 , the solution of the differential equation can be discussed. The characteristic determinant equation is that

$$
|J(Q)-\lambda E|=\left|\begin{array}{cccc}
\frac{\partial f_{1}}{\partial \Delta T_{1}}-\lambda & \frac{\partial f_{1}}{\partial \Delta T_{2}} & \frac{\partial f_{1}}{\partial \Delta T_{3}} & \frac{\partial f_{1}}{\partial \Delta T_{4}} \\
\frac{\partial f_{2}}{\partial \Delta T_{1}} & \frac{\partial f_{2}}{\partial \Delta T_{2}}-\lambda & \frac{\partial f_{2}}{\partial \Delta T_{3}} & \frac{\partial f_{2}}{\partial \Delta T_{4}} \\
\frac{\partial f_{3}}{\partial \Delta T_{1}} & \frac{\partial f_{3}}{\partial \Delta T_{2}} & \frac{\partial f_{3}}{\partial \Delta T_{3}}-\lambda & \frac{\partial f_{3}}{\partial \Delta T_{4}} \\
\frac{\partial f_{4}}{\partial \Delta T_{1}} & \frac{\partial f_{4}}{\partial \Delta T_{2}} & \frac{\partial f_{4}}{\partial \Delta T_{3}} & \frac{\partial f_{4}}{\partial \Delta T_{4}}-\lambda
\end{array}\right|=0 .
$$

Combining equations (128) (131), we can obtain the following expression:

$$
\begin{array}{cccc}
-\frac{n\left(\Delta T_{01}-\Delta T_{02}\right)}{2 A}-n A-\lambda & \frac{n \alpha\left(\Delta T_{01}-\Delta T_{02}\right)}{2 A}+n A & 0 & 0 \\
-\frac{n\left(\Delta T_{02}-\Delta T_{04}\right)}{2 A} & \frac{n \alpha\left(\Delta T_{02}-\Delta T_{04}\right)}{2 A}-n A-\lambda & 0 & n A \\
-\frac{\xi n\left(\Delta T_{03}-\Delta T_{01}\right)}{2 A}+\xi n A & \frac{\xi n \alpha\left(\Delta T_{03}-\Delta T_{01}\right)}{2 A} & -\xi n A-\lambda & 0 \\
-\frac{\xi n \Delta T_{04}}{2 A} & \frac{\xi n \alpha \Delta T_{04}}{2 A} & 0 & -\xi n A-\lambda
\end{array} \mid=0,
$$


Combining equations (153) (156), we can obtain characteristic roots as follows:

$$
\lambda_{1}=\lambda_{2}=-\xi n A=-\xi n \sqrt{\Delta T_{01}-\alpha \Delta T_{02}}=-\xi n^{2 / 3} E_{1}^{1 / 3} .
$$

Two other eigenvalues can be obtained according to the quadratic formula:

$$
\lambda_{3,4}=\frac{-b \pm \sqrt{b^{2}-4 a c}}{2 a}=\frac{-(n B / 2 A)-2 n A \pm \sqrt{[(n B / 2 A)+2 n A]^{2}-4 n^{2}\left[(B / 2)+A^{2}\right]}}{2}
$$

where

$$
\left\{\begin{array}{l}
a=1, \\
b=\frac{n B}{2 A}+2 n A=\frac{5 n^{2 / 3} E_{1}^{1 / 3}}{2}, \\
c=n^{2}\left[\frac{B}{2}+A^{2}\right]=\frac{3 n^{4 / 3} E_{1}^{2 / 3}}{2}, \\
\Delta=b^{2}-4 a c=\frac{25 n^{4 / 3} E_{1}^{2 / 3}}{4}-6 n^{4 / 3} E_{1}^{2 / 3}=\frac{n^{4 / 3} E_{1}^{2 / 3}}{4} .
\end{array}\right.
$$

It is easy to know that $\lambda_{1,2}$ are always real number $\lambda_{1}=\gamma_{1}=\lambda_{2}=\gamma_{2}$. Owing to $E_{1}>0$ and $n>0$, from equation (157), we know that $\lambda_{1,2}$ are always less than 0 ; from equation (159), we know that $\Delta>0$, and thus $\lambda_{3}$ and $\lambda_{4}$ are always real numbers $\lambda_{3}=\gamma_{3}$ and $\lambda_{4}=\gamma_{4}$. Combining equations (158) and (159), we can obtain that $\lambda_{3}=-n^{2 / 3} E_{1}^{1 / 3}$ and $\lambda 4=-\left(\left(3 n^{2 / 3} E_{1}^{1 / 3}\right) / 2\right)$. Owing to $E_{1}>0$ and $n>0$, we can know that $\lambda_{3}$ and $\lambda_{4}$ are less than 0 . In conclusion, four eigenvalues $\lambda_{1}, \lambda_{2}, \lambda_{3}$, and $\lambda_{4}$ are less than 0 , and it is clear that the equilibrium point is stable in realization 2 .

In brief, for the scenario 3 of one heat source at the bottom of the building, because the heat can enter either the left or the right side, two steady states always exist for this scenario. The parameter $\alpha$ will not affect the stability and existence of solution of buoyancy ventilation of this building configuration.

\section{Model Validation}

Reference [35] showed the comparison results with [34], which is the literature studying the structure of one heat source at the bottom with two adiabatic tunnels. In order to verify the validity and accuracy of our model, similarly, we compared the scenario 3 results with those of [34]. The outdoor temperature is $288 \mathrm{~K}$, the air density $1.225 \mathrm{~kg} / \mathrm{m}^{3}, C_{p}$ $1.0 \mathrm{~kJ} /(\mathrm{kg} \cdot \mathrm{K})$, heat source $1 \mathrm{~kW}, H_{1} 5.5 \mathrm{~m}, H_{2} 5.5 \mathrm{~m}, S_{1+2+3+4}$ $37.2933 \mathrm{~kg}^{-1} / \mathrm{m}^{-1}$, and gravity acceleration $\mathrm{g} 9.81 \mathrm{~m} / \mathrm{s}^{2}$.

The comparison in temperature difference between the proposed model and the validated results from [34] is illustrated in Figure 2(a). The comparison in flow rate between the proposed model and the validated results from [34] is illustrated in Figure 2(b). In [35], we know the maximum relative error for the temperature difference is $13.2 \%$ and the maximum relative error for the flow rate is $15.9 \%$, when the strength of the local heat source is $100 \mathrm{~W}$. While in our results, the maximum relative error for the temperature difference is $2.16 \%$ and the maximum relative error for the flow rate is $3.23 \%$. These show that our results are closer to the CFD simulation results in [34] than in [35], which means that the proposed 4-zone model in our paper is more reasonable than 2-zone model in [35].

Referring to method of [35], we rectify our model and obtain the rectified results of temperature and flow rate. Here, we give rectified results in zone 2 for the realization 1 . Tunnel $H_{1}$ on the left remains unchanged to $5.5 \mathrm{~m}$, and tunnel $\mathrm{H}_{2}$ on the right is subtracted from room height. In order to compare with $[34,35]$, the height of the room is $0.5 \mathrm{~m}$, so after adjustment, $H_{2}=5 \mathrm{~m}$. After improvement, the maximum relative error of flow rate is $1.0 \%$ and the maximum relative error of temperature is $1.58 \%$ when our results are compared with the ones in [34]. While in [35], the maximum relative error of flow rate is $12.31 \%$ and the maximum relative error of temperature is $10.39 \%$. Therefore, the results of the model we proposed are closer to the CFD results of [34].

\section{Representation of Phase Portraits}

We compare the formation process of the system from different initial conditions to the steady state under different control parameter $a$ by means of the vector field and phase portrait. Based on the analysis of stability of equilibrium points for scenario 1 , we fix control parameter $k$ to 10 and four typical values of $\alpha$ are selected, which are $0.05,0.5,1$, and 2 . We make phase portrait and vector field for scenario 1 $(k>0)$ through MATLAB, and the results are shown in Figure 3. Figure 3(a) denotes the phase portrait and vector field for $k=10$ and $\alpha=0.05$ of realization 2; Figure 3(b) denotes phase portrait and vector field for $k=10$ and $\alpha=0.5$, where the red point and blue point denote the equilibrium point of realization 1 and realization 2 , respectively. Figures 3(c) and 3(d) display the vector field and phase portrait of realization 1 and realization 2 , respectively, when the control parameter $\alpha$ is equal to 1 . The red dot in Figure 3(c) represents the stable equilibrium point of realization 1 , and the initial condition around the equilibrium point converges to stable equilibrium point. The blue dot in Figure 3(d) represents the unstable equilibrium point of realization 2 , and the initial condition around the unstable 


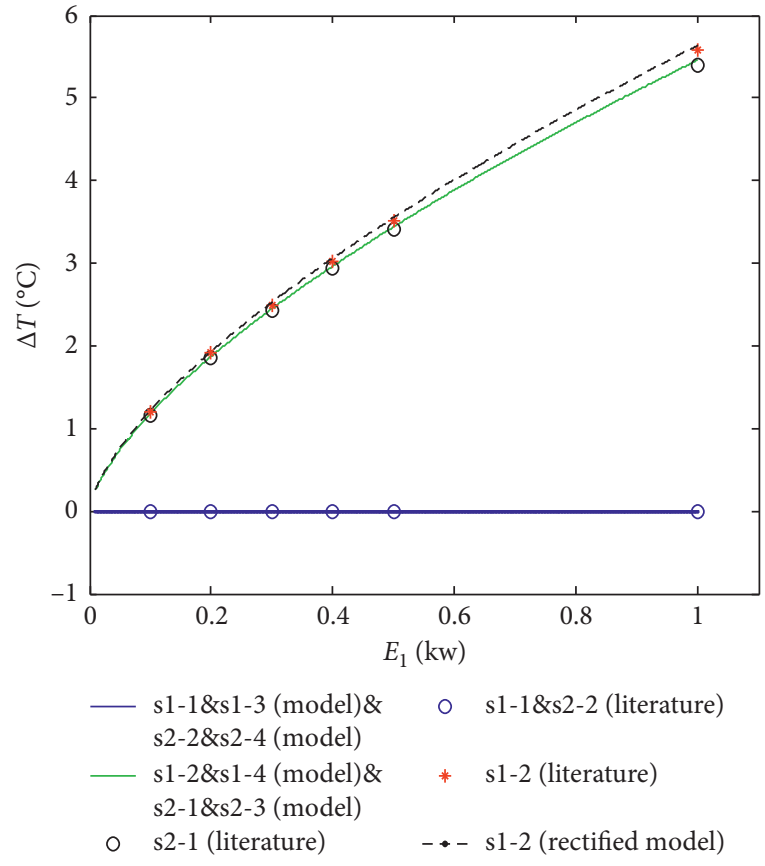

(a)

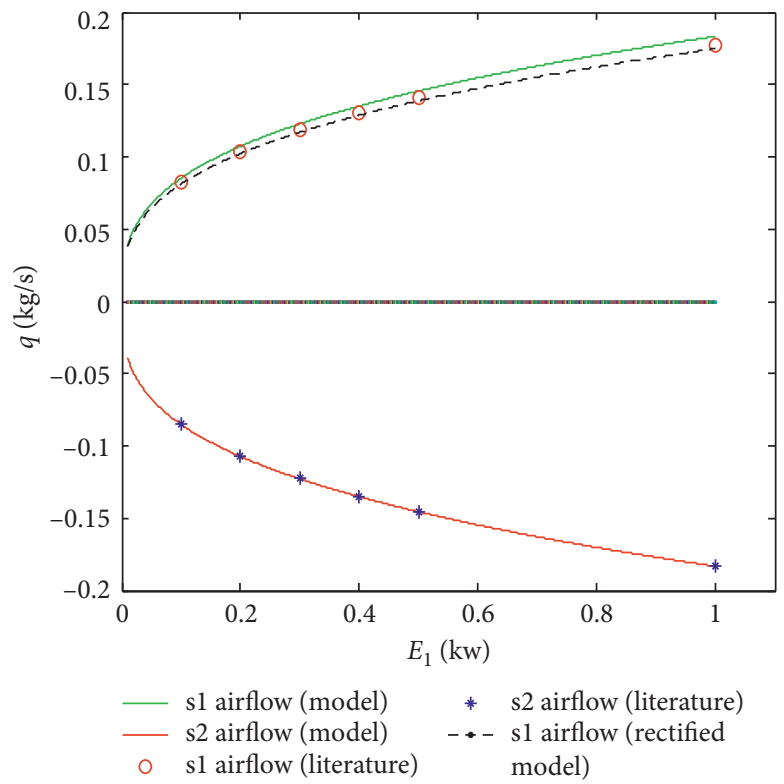

(b)

Figure 2: Modeled results validation. (a) Temperature comparison between the four-zone model and previous CFD results. (b) Mass flow rates comparison between the four-zone model and previous CFD results (S1 indicates status1/realization 1; 1, 2, etc. indicates zone serial number).

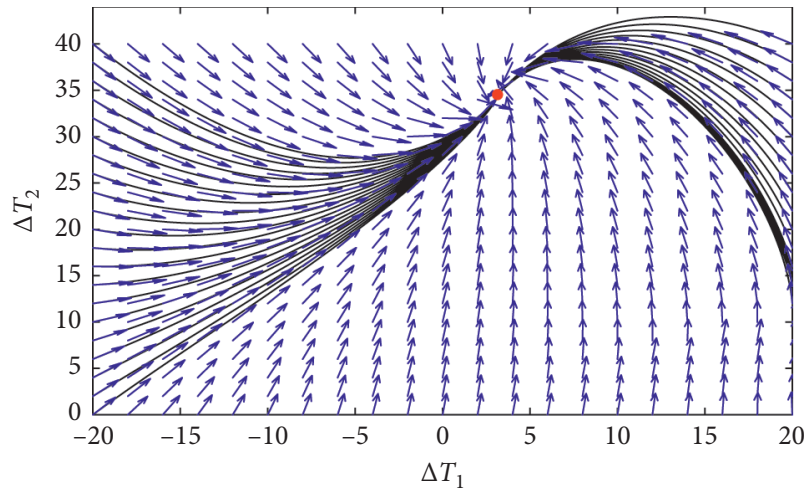

(a)

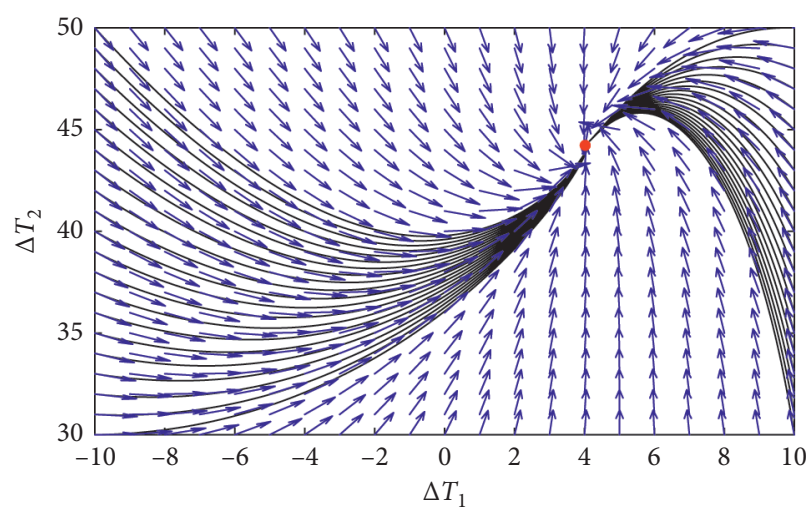

(c)

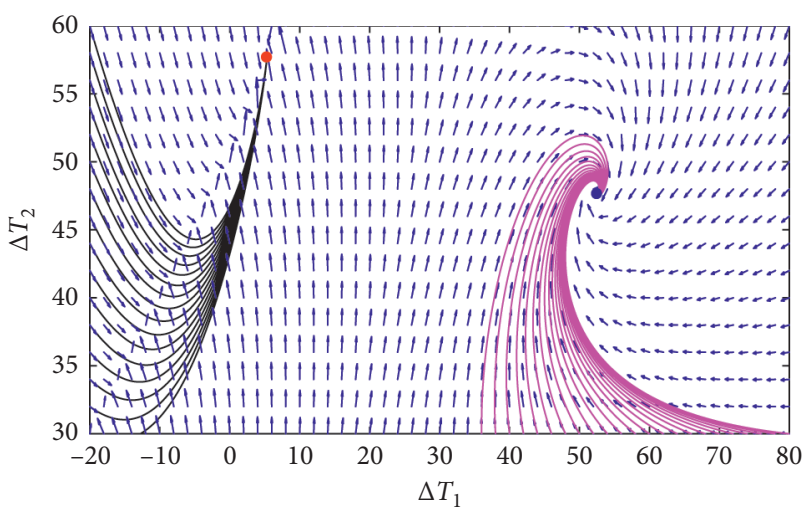

(b)

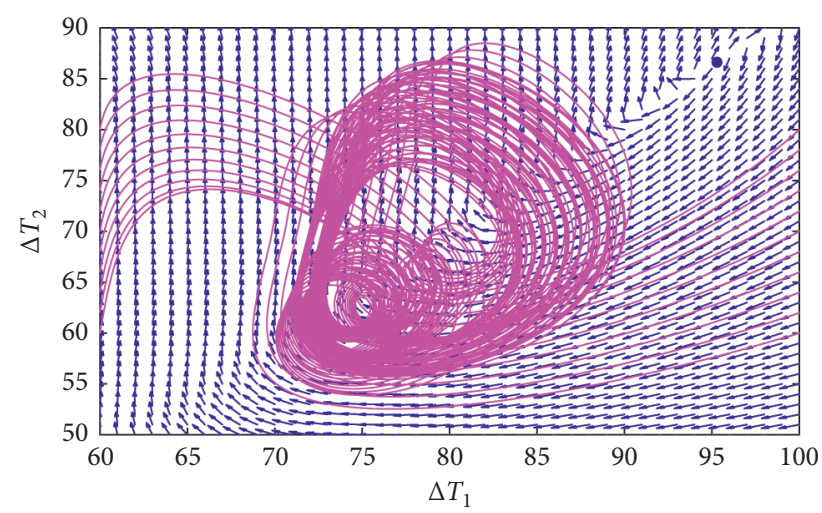

(d)

FIgUre 3: Continued. 


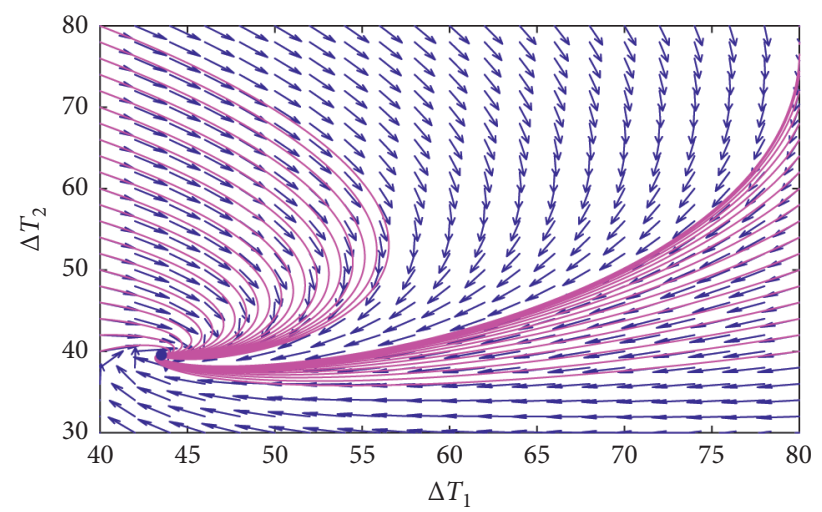

(e)

Figure 3: Phase portrait and vector field for scenario $1(k>0)$ : (a) $k=10$ and $\alpha=0.05$ of realization 2; (b) $k=10$ and $\alpha=0.5$, where the red point and blue point denote the equilibrium point of realization 1 and realization 2, respectively; (c) $k=10$ and $\alpha=1$ of realization 1 ; (d) $k=10$ and $\alpha=1$ of realization 2 ; (e) $k=10$ and $\alpha=2$ of realization 1 .

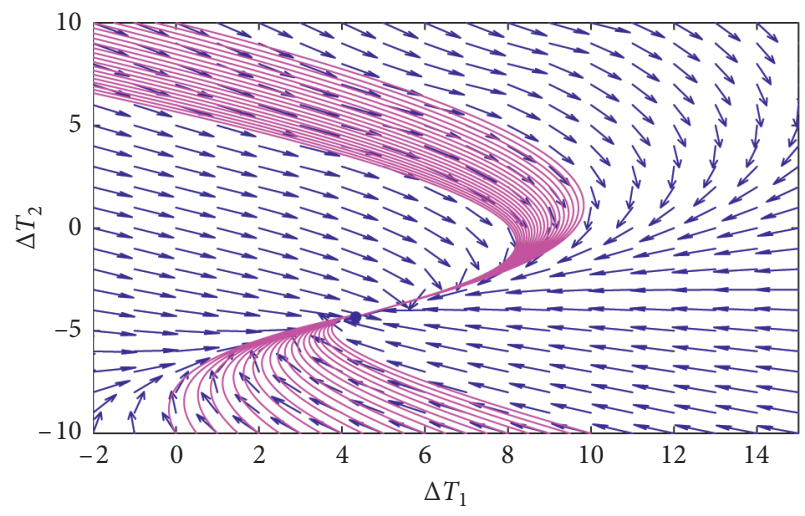

(a)

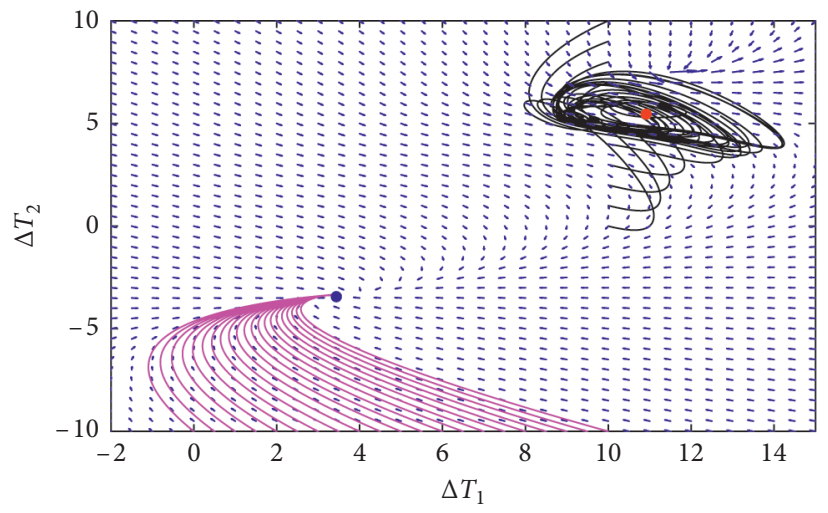

(b)

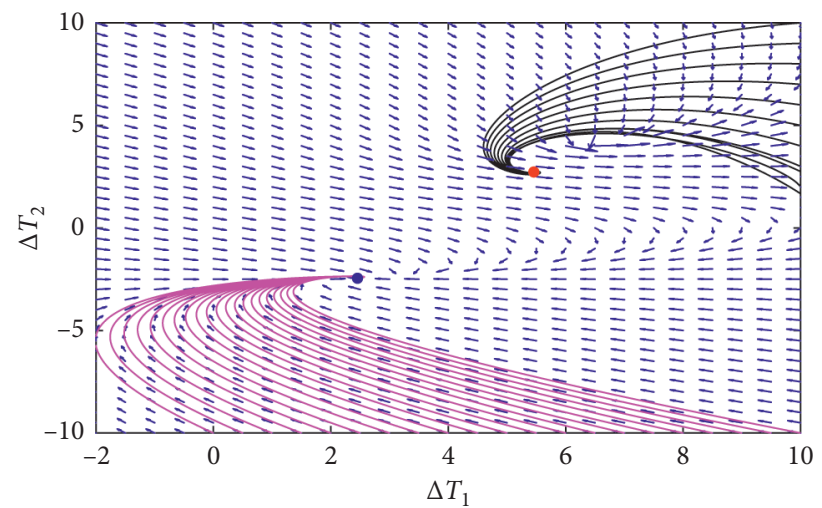

(c)

Figure 4: Phase portrait and vector field for scenario $1(k<0)$. (a) $k=-0.5$ and $\alpha=1$ of realization 2 ; (b) $k=-0.5$ and $\alpha=3$, where the red dot indicates the equilibrium point of realization 1 and the blue dot represents the equilibrium point of realization 2 ; (c) $k=-0.5$ and $\alpha=10$, where the red dot indicates the equilibrium point of realization 1 and the blue dot represents the equilibrium point of realization 2 .

equilibrium point forms the periodic motion. Figure 3(e) denotes the phase portrait and vector field for $k=10$ and $\alpha=2$ of realization 1. In Figure 3(e), there is only one stable equilibrium point of realization 1 , and all different initial states converge to the same steady state.
Based on the analysis of stability of equilibrium points for scenario 1, we fix control parameter $k$ to -0.5 , and three typical values of $\alpha$ are selected, which are 1,3 , and 10 . We make phase portrait and vector field for scenario $1(k<0)$, and the results are shown in Figure 4. Figure 4(a) denotes the 


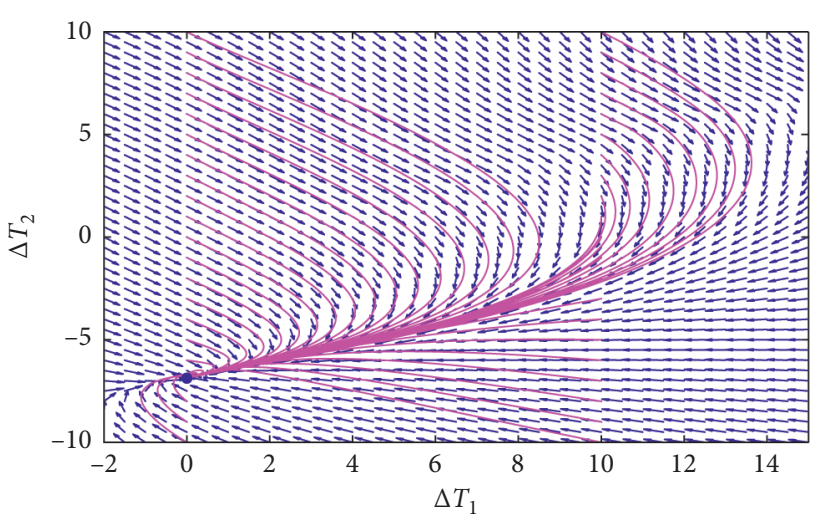

(a)

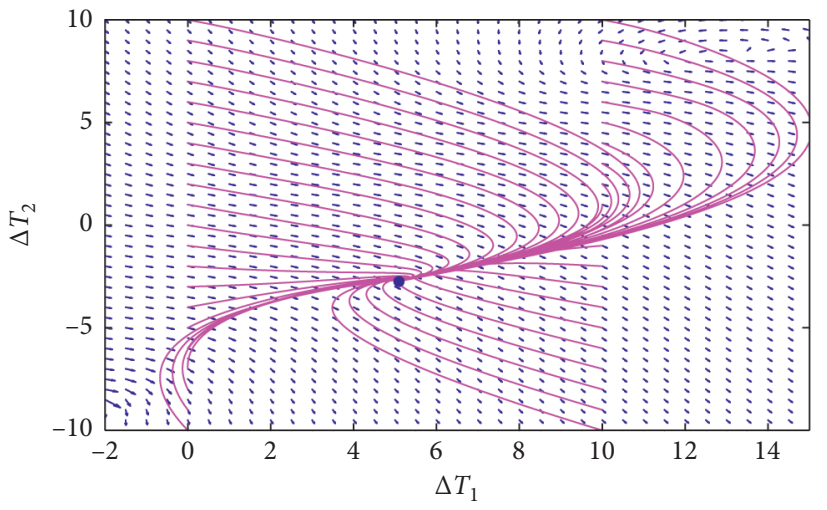

(c)

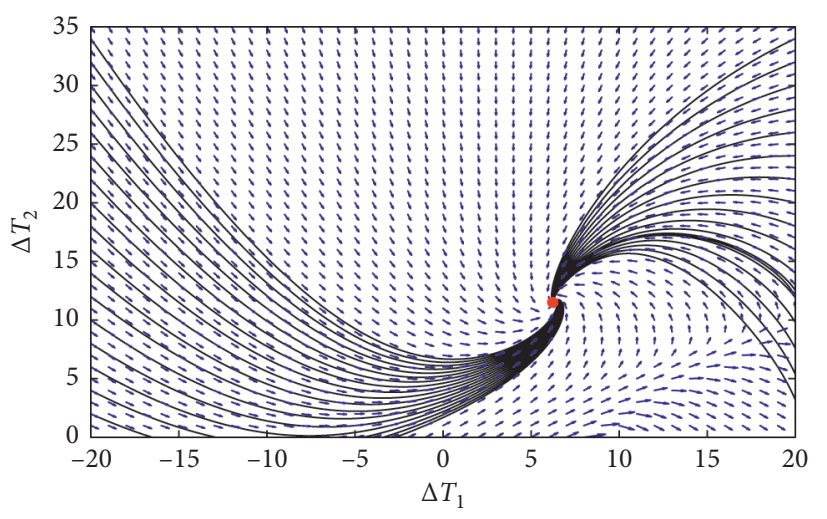

(e)

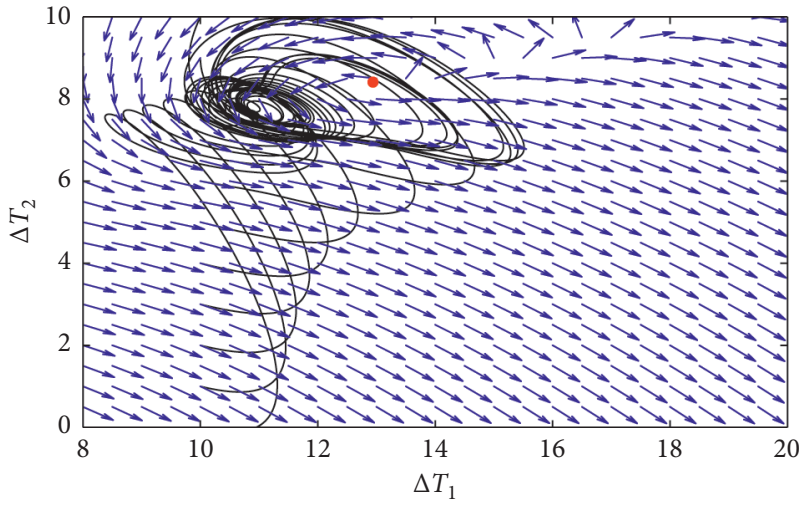

(b)

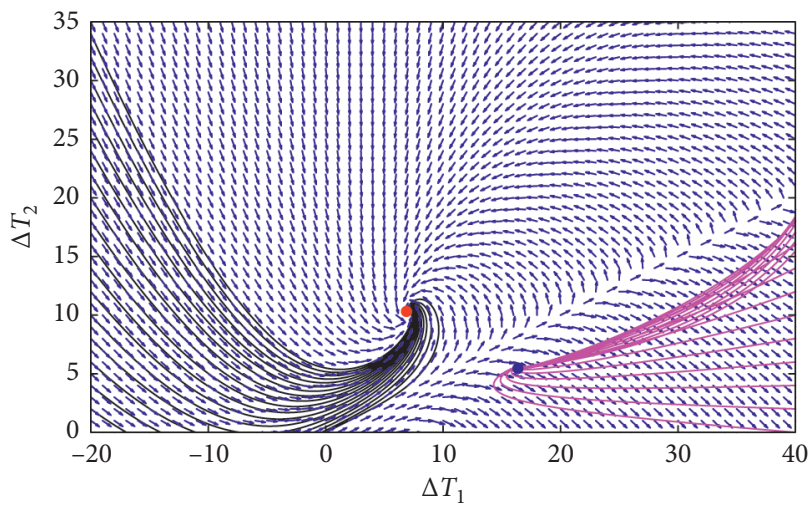

(d)

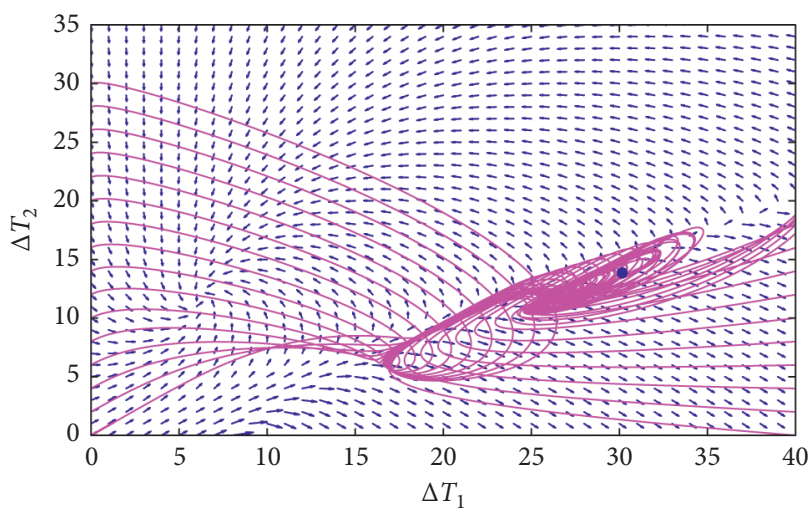

(f)

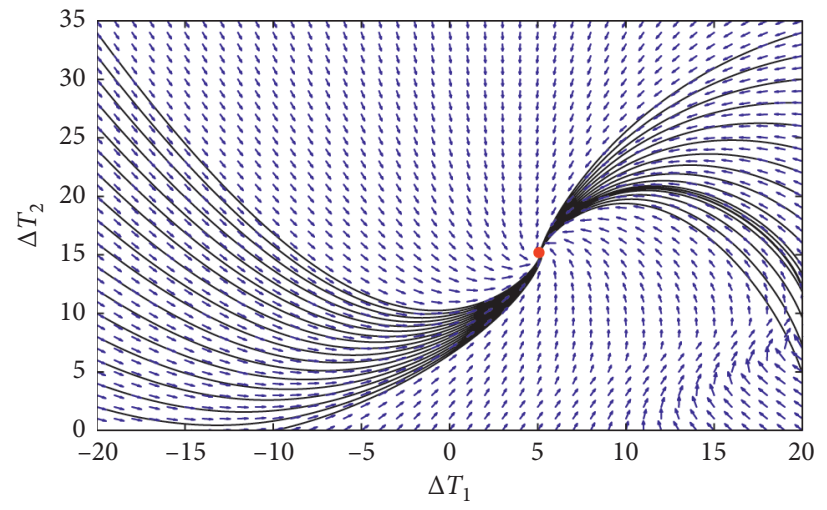

(g)

Figure 5: Phase portrait and vector field for scenario 2. (a) $\alpha=2$ and $k=-1$ of realization 2; (b) $\alpha=2$ and $k=-0.35$ of realization 1 ; (c) $\alpha=2$ and $k=-0.35$ of realization 2 ; (d) $\alpha=2$ and $k=0.5$, where the red dot indicates the equilibrium point of realization 1 and the blue dot represents the equilibrium point of realization 2; (e) $\alpha=2$ and $k=0.85$ of realization 1 ; (f) $\alpha=2$ and $k=0.85$ of realization 2 ; (g) $\alpha=2$ and $k=2$ of realization 1 . 
phase portrait and vector field for $k=-0.5$ and $\alpha=1$ of realization 2. In Figure 4(a), only one stable equilibrium point appeared, and all different initial states converge to the same steady state. Figure 4(b) denotes the phase portrait and vector field for $k=-0.5$ and $\alpha=3$. In Figure 4(b), the red dot represents the unstable equilibrium point of realization 1 , and the initial condition around the unstable equilibrium point converges to periodic motion. And, the blue dot represents stable equilibrium point of realization 2 , and the initial conditions around the stable equilibrium point converge to the equilibrium point. Figure 4 (c) denotes phase portrait and vector field for $k=-0.5, \alpha=10$. The red dot and blue dot in Figure 4(c) represent the stable equilibrium point of realization 1 and realization 2 , respectively. The initial condition around the red dot converges to the stable equilibrium point of realization 1 , and the initial condition around the blue dot converges to the equilibrium point of realization 2 .

Based on the analysis of stability of equilibrium points for scenario 2, we fixed control parameter $\alpha$ to 2, and five typical values of $k$ were selected, which were $-1,-0.35,0.5$, 0.85 , and 2 . We make phase portrait and vector field for scenario 2, and the results are shown in Figure 5. In Figure 5(a), only one stable equilibrium point of realization 2 appeared, when $k$ is equal to -1 , and all different initial states converge to the same steady state. Figures 5(b) and 5(c) display the vector field and phase portrait of realization 1 and realization 2 , respectively, when the control parameter $k$ is equal to -0.35 . The red dot in Figure 5(b) represents the unstable equilibrium point of realization 1 , and the initial condition around the unstable equilibrium point forms the periodic motion. The blue dot in Figure 5(c) represents the stable equilibrium point of realization 2 , and the initial condition around the equilibrium point converges to stable equilibrium point. Figure 5(d) denotes the phase portrait and vector field for $\alpha=2$ and $k=0.5$, where the red dot indicates the equilibrium point of realization 1 and the blue dot represents the equilibrium point of realization 2 . Figures 5(e) and 5(f) display the vector field and phase portrait of realization 1 and realization 2 , respectively, when the control parameter $k$ is equal to 0.85 . The red dot in Figure 5(e) represents the stable equilibrium point of realization 1 , and the initial condition around the equilibrium point converges to stable equilibrium point. The blue dot in Figure 5(f) represents the unstable equilibrium point of realization 2 , and the initial condition around the unstable equilibrium point forms the periodic motion. In Figure 5(g), there is only one stable equilibrium point of realization 1 , and all different initial states converge to the same steady state.

\section{Conclusions}

Nonlinear dynamical analysis was performed to study the buoyancy ventilation of a typical four-zone underground building. A new model with four zones that described the buoyancy ventilation of the underground buildings was proposed and validated by results from the previous studies. The new model contained four nonlinear ordinary differential equations. The criteria for the stability and existence of equilibrium points were derived mathematically in detail about three different scenarios. The criterion for scenario 1 ( $k$ was fixed and control $\alpha$ ) is summarized in Tables 1 and 2, which correspond $k>0$ and $k<0$, respectively; the criterion for scenario 2 ( $\alpha$ was fixed and control $\kappa$ ) is summarized in Table 3. Two stable equilibrium points existed in scenario 3 (one heat source at the bottom of the building and control $\alpha$ ). Finally, the phase portraits and vector field diagrams obtained by applying the fourth-order Runge-Kutta method are given.

\section{Nomenclature}

$q_{1} \sim q_{4}: \quad$ Mass flow rate at zones $1 \sim 4(\mathrm{~kg} / \mathrm{s})$

$T_{1} \sim \mathrm{T}_{4}: \quad$ Air temperature at zones $1 \sim 4(\mathrm{~K})$

$T_{a}$ : $\quad$ Outdoor air temperature $(\mathrm{K})$

$\mathrm{S}_{1} \sim \mathrm{S}_{4}$ : $\quad$ Coefficient of mass flow impedance at zones $1 \sim 4$

$M_{1} \sim M_{4}$ : Thermal mass at zones $1 \sim 4(\mathrm{~kg})$

$E_{1}$ : Total heat gain at zone $1(\mathrm{~kW})$

$E_{2}$ : $\quad$ Total heat gain at zone $2(\mathrm{~kW})$

$C_{p}: \quad$ Specific heat of air $(\mathrm{kJ} /(\mathrm{kg} \cdot \mathrm{K}))$

$t: \quad$ Time $(\mathrm{s})$

$\xi$ : $\quad$ Mass ratio between zones 1 or 2 and

zones 3 or 4

g: $\quad$ Gravitational acceleration $\left(\mathrm{m} / \mathrm{s}^{2}\right)$

$H_{1}$ : Height of zone 3 (left tunnel) (m)

$\mathrm{H}_{2}$ : $\quad$ Height of zone 4 (right tunnel) (m)

$k: \quad$ Heat ratio between two zones $E_{2} / E_{1}$

$\Delta T_{1} \sim \Delta T_{4}$ : Temperature difference between indoor and outdoor air at zones $1 \sim 4\left({ }^{\circ} \mathrm{C}\right)$

$\alpha: \quad$ Height ratio between two zones $\mathrm{H}_{2} / \mathrm{H}_{1}$

$\Delta T_{10} \sim \Delta T_{40}$ : Temperature difference between indoor and outdoor air at zones $1 \sim 4$ in steady state $\left({ }^{\circ} \mathrm{C}\right)$

$\rho_{0}: \quad$ Ambient air density $\left(\mathrm{kg} / \mathrm{m}^{3}\right)$

$J(Q): \quad$ Jacobian matrix of equilibrium point $Q$

$\lambda$ : $\quad$ Eigenvalue of coefficient matrix.

\section{Data Availability}

All data used to support the findings of this study are available from the corresponding author upon request.

\section{Conflicts of Interest}

The authors declare that they have no conflicts of interest.

\section{Acknowledgments}

The work was supported by the National Natural Science Foundation of China (no. 51378184)

\section{References}

[1] W. Yao, C. Wang, Y. Sun, C. Zhou, and H. Lin, "Synchronization of inertial memristive neural networks with timevarying delays via static or dynamic event-triggered control," Neurocomputing, vol. 404, pp. 367-380, 2020. 
[2] W. Yao, C. Wang, J. Cao, Y. Sun, and C. Zhou, "Hybrid multisynchronization of coupled multistable memristive neural networks with time delays," Neurocomputing, vol. 363, pp. 281-294, 2019.

[3] C. Zhou, C. H. Wang, Y. C. Sun, and W. Yao, "Weighted sum synchronization of memristive coupled neural networks," Neurocomputing, vol. 403, pp. 225-232, 2020.

[4] C. Li, K. Qian, S. He, H. Li, and W. Feng, "Dynamics and optimization control of a robust chaotic map," IEEE Access, vol. 7, pp. 160072-160081, 2019.

[5] F. Yu, L. Liu, H. Shen et al., "Multistability analysis, coexisting multiple attractors and FPGA implementation of Yu-Wang four-wing chaotic system," Mathematical Problems in Engineering, vol. 2020, Article ID 7530976, 2020.

[6] F. Yu, L. Liu, B. Shen et al., "Dynamic analysis, circuit design and synchronization of a novel 6D memristive four-wing hyperchaotic system with multiple coexisting attractors," Complexity, vol. 2020, Article ID 5904607, 17 pages, 2020.

[7] Z. Wen, Z. Li, and X. Li, "Transient MMOs in memristive chaotic system via tiny perturbation," Electronics Letters, vol. 56, no. 2, pp. 78-80, 2020.

[8] Q. Zhao, C. Wang, and X. Zhang, "A universal emulator for memristor, memcapacitor, and meminductor and its chaotic circuit," Chaos: An Interdisciplinary Journal of Nonlinear Science, vol. 29, no. 1, p. 13141, 2019.

[9] L. Y. Wang, K. H. Sun, Y. X. Peng, and S. B. He, "Chaos and complexity in a fractional-order higher-dimensional multicavity chaotic map," Chaos Solitons \& Fractals, vol. 131, Article ID 109488, 2020.

[10] X. Zhang, C. Wang, W. Yao, and H. Lin, "Chaotic system with bondorbital attractors," Nonlinear Dynamics, vol. 97, no. 4, pp. 2159-2174, 2019.

[11] Q. Deng and C. Wang, "Multi-scroll hidden attractors with two stable equilibrium points," Chaos: An Interdisciplinary Journal of Nonlinear Science, vol. 29, no. 9, p. 093112, 2019.

[12] Q. L. Deng, C. H. Wang, and L. M. Yang, "Four-wing hidden attractors with one stable equilibrium point," International Journal of Bifurcation and Chaos, vol. 30, no. 6, Article ID 2050086, 2020.

[13] M. J. Zhou and C. H. Wang, "A novel image encryption scheme based on conservative hyperchaotic system and closed-loop diffusion between blocks," Signal Processing, vol. 171, Article ID 107484, 2020.

[14] S. C. Wang, C. H. Wang, and C. Xu, "An image encryption algorithm based on a hidden attractor chaos system and the Knuth-Durstenfeld algorithm," Optics and Lasers in Engineering, vol. 128, Article ID 105995, 2020.

[15] C. Xu, J. R. Sun, and C. H. Wang, "An image encryption algorithm based on random walk and hyperchaotic systems," International Journal of Bifurcation and Chaos, vol. 30, no. 4, Article ID 2050060, 2020.

[16] K. Nitta, "Variety modes and chaos in smoke ventilation by ceiling chamber system," in Proceedings of the 6th International IBPSA Conference, Kyoto, Japan, 1999.

[17] G. R. Hunt and P. P. Linden, "The fluid mechanics of natural ventilation-displacement ventilation by buoyancy-driven flows assisted by wind," Building and Environment, vol. 34, no. 6, pp. 707-720, 1999.

[18] Y. Li, A. Delsante, Z. Chen et al., "Some examples of solution multiplicity in natural ventilation," Building and Environment, vol. 36, no. 7, pp. 851-858, 2001.

[19] J. Yuan and L. R. Glicksman, "Multiple steady states in combined buoyancy and wind driven natural ventilation: the conditions for multiple solutions and the critical point for initial conditions," Building and Environment, vol. 43, no. 1, pp. 62-69, 2008.

[20] C. Gladstone and A. W. Woods, "On buoyancy-driven natural ventilation of a room with a heated floor," Journal of Fluid Mechanics, vol. 441, pp. 293-314, 2001.

[21] T. Chenvidyakarn and A. Woods, "Multiple steady states in stack ventilation," Building and Environment, vol. 40, no. 3, pp. 399-410, 2005.

[22] J. Gong and Y. Li, "Smoke flow bifurcation due to opposing buoyancy in two horizontally connected compartments," Fire Safety Journal, vol. 59, pp. 62-75, 2013.

[23] D. Yang, P. Li, H. Duan et al., "Multiple patterns of heat and mass flow induced by the competition of forced longitudinal ventilation and stack effect in sloping tunnels," International Journal of Thermal Sciences, vol. 138, pp. 35-46, 2019.

[24] P. Heiselberg, Y. Li, A. Andersen, M. Bjerre, and Z. Chen, "Experimental and CFD evidence of multiple solutions in a naturally ventilated building," Indoor Air, vol. 14, no. 1, pp. 43-54, 2004.

[25] B. Lishman and A. W. Woods, "On transitions in natural ventilation flow driven by changes in the wind," Building and Environment, vol. 44, no. 4, pp. 666-673, 2009.

[26] J. Yuan and L. R. Glicksman, "Transitions between the multiple steady states in a natural ventilation system with combined buoyancy and wind driven flows," Building and Environment, vol. 42, no. 10, pp. 3500-3516, 2007.

[27] E. Pulat and H. A. Ersan, "Numerical simulation of turbulent airflow in a ventilated room: inlet turbulence parameters and solution multiplicity," Energy and Buildings, vol. 93, pp. 227-235, 2015.

[28] F. Durrani, M. J. Cook, and J. J. McGuirk, "Evaluation of LES and RANS CFD modelling of multiple steady states in natural ventilation," Building and Environment, vol. 92, pp. 167-181, 2015.

[29] Z. D. Chen and Y. Li, "Buoyancy-driven displacement natural ventilation in a single-zone building with three-level openings," Building and Environment, vol. 37, no. 3, pp. 295-303, 2002.

[30] L. Yang, Y. Li, P. Xu, and G. Zhang, "Nonlinear dynamic aalysis of natural ventilation in a two-zone building: Part B-CFD simulations," HVAC\&R Research, vol. 12, no. 2, pp. 257-278, 2006.

[31] L. Yang, P. Xu, and Y. Li, "Nonlinear dynamic analysis of natural ventilation in a two-zone building: part A-theoretical analysis," HVAC\&R Research, vol. 12, no. 2, pp. 231-255, 2006.

[32] Y. Li, P. Xu, H. Qian, Q.-H. Deng, and J. Wu, "Flow bifurcation due to opposing buoyancy in two vertically connected open cavities," International Journal of Heat and Mass Transfer, vol. 49, no. 19-20, pp. 3298-3312, 2006.

[33] D. Yang, Y. Liu, C. Zhao, and S. Mao, "Multiple steady states of fire smoke transport in a multi-branch tunnel: theoretical and numerical studies," Tunnelling and Underground Space Technology, vol. 61, pp. 189-197, 2017.

[34] Y. Liu, Y. Xiao, G. Augenbroe et al., "The formation of multisteady-states of buoyancy ventilation in underground building," Tunnelling and Underground Space Technology, vol. 82, pp. 613-626, 2018.

[35] Y. Liu, Y. Xiao, and J. L. Chen, "Nonlinear dynamic analysis of solution multiplicity of buoyancy ventilation in a typical underground structure," Building and Environment, vol. 171, Article ID 106674, 2020. 